Bull. Soc. math. France

134 (3), 2006, p. 417-445

\title{
UNE APPROCHE HILBERTIENNE DE L'HYPOTHÈSE DE RIEMANN GÉNÉRALISÉE
}

\author{
PAR ANNE DE Roton
}

\begin{abstract}
RÉSumÉ. - En généralisant dans [9] le théorème de Beurling et Nyman à la classe de Selberg, nous avons reformulé l'hypothèse de Riemann généralisée en terme d'un problème d'approximation. Nous poursuivons ici ce travail de généralisation par l'étude d'une distance liée à ce problème. Nous donnons une minoration de cette distance, ce qui constitue une extension du travail de Burnol [7] et de celui de Báez-Duarte, Balazard, Landreau et Saias [2], travail qui concernait la fonction $\zeta$ de Riemann et que nous étendons aux fonctions de la classe de Selberg.

ABstRaCt (An hilbertian approach of the generalised Riemann hypothesis)

In [9], we generalised Beurling and Nyman's criterion to functions in Selberg's class and therefore gave a formulation of the generalised Riemann hypothesis as an approximation problem. We give a lower bound for the distance involved in this problem. This is an extension of the papers [7] and [2], in which the Riemann zeta function was studied whereas we study any function in Selberg's class.
\end{abstract}

\section{Présentation des résultats; historique}

Le théorème de Beurling et Nyman (cf. [4]) reformule l'hypothèse de Riemann pour la fonction $\zeta$ en terme d'un problème d'approximation. On pourra consulter [3] pour un nouvel éclairage de ce théorème.

Texte reçu le 4 avril 2005, accepté le 26 octobre 2005.

Anne de Roton, Institut Élie Cartan, UMR 7502, Nancy-Université, BP 239, 54506 Vandœuvre-lès-Nancy (France). • E-mail : deroton@iecn.u-nancy.fr

Classification mathématique par sujets (2000). - 11M41.

Mots clefs. - Hypothèse de Riemann généralisée, classe de Selberg, opérateurs, transformée de Mellin, distance hilbertienne. 
Les travaux récents de Báez-Duarte, Balazard, Landreau et Saias [2] et Burnol [7] ont permis de poursuivre l'étude de ce problème d'approximation, toujours pour la fonction $\zeta$ de Riemann.

Nous avons étendu dans [9] le théorème de Beurling et Nyman aux fonctions de la classe de Selberg. Nous allons dans cet article étendre le résultat de Burnol, qui améliore le résultat de [2], à cette même classe de fonctions.

Pour cela, nous construirons des vecteurs «presque orthogonaux » (nous préciserons plus loin cette notion) indexés par les zéros de la fonction de la classe de Selberg considérée. Afin d'obtenir un résultat analogue à celui de Burnol, nous prendrons en compte la multiplicité des zéros.

Commençons par rappeler la définition de la classe de Selberg $S$, introduite en 1989 dans [?], et qui est conjecturalement l'ensemble des fonctions $L$ de formes automorphes.

DÉfinition 1. - On dira qu'une fonction $F$ appartient à la classe de Selberg $S$ si elle vérifie les conditions suivantes :

1) pour Re $s>1, F(s)=\sum_{n=1}^{\infty} a_{n} n^{-s}$ est une série de Dirichlet absolument convergente;

2) il existe un entier naturel $m$ tel que $(s-1)^{m} F(s)$ soit une fonction entière d'ordre fini;

3) la fonction $F$ satisfait une équation fonctionnelle de la forme :

$$
\Phi(s)=\omega \overline{\Phi(1-\bar{s})} \quad \text { où } \quad \Phi(s)=Q^{s} \Delta(s) F(s),
$$

avec $\Delta(s)=\prod_{j=1}^{r} \Gamma\left(\lambda_{j} s+\mu_{j}\right), \lambda_{j}>0, \operatorname{Re} \mu_{j} \geq 0, Q>0$ et $|\omega|=1 ;$

4) pour tout $\varepsilon>0, a_{n}=O\left(n^{\varepsilon}\right)$;

5) pour Re $s$ assez grand, $\log F(s)=\sum_{n=1}^{+\infty} b_{n} n^{-s}$ où $b_{n}=0$ si $n$ n'est pas une puissance d'un nombre premier et $b_{n}=O\left(n^{\theta}\right)$ pour un $\theta<\frac{1}{2}$.

Notations 1. - On notera :

- $d=2 \sum_{j=1}^{r} \lambda_{j}$ le degré de $F$,

- $\mu=\sum_{j=1}^{r}\left(\mu_{j}-\frac{1}{2}\right)$.

- $\bar{f}$ la fonction $s \mapsto \overline{f(\bar{s})}$.

Parmi les zéros d'une fonction $F$ de la classe de Selberg, on distinguera :

- les zéros de la forme $-\left(n+\mu_{j}\right) / \lambda_{j}, n \in \mathbb{N}, j \in[1, r]$, que l'on appelera zéros triviaux de $F$;

- les zéros de $F$ de partie réelle égale à $\frac{1}{2}$ sont appelés zéros critiques de $F$;

- la droite d'équation $\operatorname{Re} s=\frac{1}{2}$ sera appelée droite critique.

- la partie du plan constituée des points de partie réelle comprise entre 0 et 1 sera appelée bande critique.

Conjecture 1. - Les zéros non triviaux de F sont de partie réelle égale à $\frac{1}{2}$.

TOME $134-2006-\mathrm{N}^{\mathrm{O}} 3$ 
C'est ce que l'on appelle hypothèse de Riemann généralisée pour la fonction $F$ et nous la noterons désormais (HRG).

Notons qu'en utilisant la symétrie induite par l'équation fonctionnelle, cette conjecture est équivalente à la non-annulation de la fonction $F$ dans le demiplan $\operatorname{Re} s>\frac{1}{2}$.

Afin de caractériser l'hypothèse de Riemann généralisée pour une fonction $F$ de la classe de Selberg, nous avons introduit dans [9] la fonction complémentaire associée à $F$.

DÉfinition 2. - Soit $F$ une fonction de la classe de Selberg. On définit la fonction complémentaire associée à $F$ par

$$
\Psi_{F}: \mathbb{R}^{+} \longmapsto \mathbb{C}, \quad x \longmapsto \operatorname{Res}\left(\frac{x^{s}}{s} F(s), 1\right)-\sum_{n \leq x} a_{n} .
$$

On définit également la fonction $\Psi_{F}^{(1)}$ par

$$
\Psi_{F}^{(1)}(x)=\Psi_{F}\left(\frac{1}{x}\right) .
$$

Nous avons démontré dans [9] que l'hypothèse de Riemann généralisée pour une fonction $F$ de la classe de Selberg était équivalente à la densité d'un sousespace de fonctions dans $L^{2}(0,1)$. Plus précisément, pour $\lambda>0$, on définit le sous-espace de fonctions

$$
\mathcal{B}_{F}^{\lambda}=\left\{f: t \mapsto \sum_{k=1}^{n} c_{k} \Psi_{F}\left(\frac{\alpha_{k}}{t}\right), n \in \mathbb{N}, \forall k \in[1, n], c_{k} \in \mathbb{C}, \lambda \leq \alpha_{k} \leq 1\right\} .
$$

Dans le cas où $\Psi_{F}^{(1)} \in L^{2}(0,+\infty)$, on définit la quantité $D(\lambda)$ comme la distance dans $L^{2}(0,+\infty)$ entre la fonction $\chi$ indicatrice de l'intervalle $[0,1]$ et le sousespace $\mathcal{B}_{F}^{\lambda}$.

Le théorème que nous avons obtenu est le suivant :

THÉORÈme 1. - Soit F une fonction de la classe de Selberg S. Alors les assertions suivantes sont équivalentes :

1) la fonction $F$ vérifie l'hypothèse de Riemann généralisée;

2) on $a \Psi_{F}^{(1)} \in L^{2}(0,+\infty)$ et $\lim _{\lambda \rightarrow 0} D(\lambda)=0$.

Remarque 1. - Nous avons démontré que la condition $\Psi_{F}^{(1)} \in L^{2}(0,+\infty)$ était vérifée pour les fonctions de la classe de Selberg de degré strictement inférieur à 4 (cf. [10]).

Nous nous intéressons dans cet article à la fonction distance $D(\lambda)$ dans le cas où $\Psi_{F}^{(1)} \in L^{2}(0,+\infty)$. Le théorème que nous démontrons permet de majorer la vitesse de convergence de la fonction distance $D(\lambda)$. Plus précisément, nous obtenons le théorème suivant : 
THÉORÈme 2. - Soit $F$ une fonction de la classe de Selberg telle que $\Psi_{F}^{(1)}$ appartient à $L^{2}(0,+\infty)$. Alors on a

$$
\lim _{\lambda \rightarrow 0} D(\lambda) \sqrt{\log \left(\frac{1}{\lambda}\right)} \geq \sqrt{\sum_{\rho} \frac{m_{\rho}^{2}}{|\rho|^{2}}}
$$

où la somme porte sur les zéros $\rho$ de $F$ dans la bande critique et $m_{\rho}$ désigne la multiplicité du zéro $\rho$ de $F$.

Remarque 2. - La majoration, donnée dans [?], du nombre $N(T)$ de zéros $\rho$ d'une fonction de la classe de Selberg vérifiant $|\operatorname{Im} \rho|<T$ assure la convergence de la somme $\sum_{\rho} m_{\rho}^{2} /|\rho|^{2}$.

Ce résultat est l'analogue de celui de Burnol [7] pour la fonction $\zeta$. Le résultat des auteurs de [2] ne faisait pas intervenir la multiplicité des zéros de la fonction $\zeta$ et était donc moins précis. Il est d'autre part notable que seuls les zéros critiques interviennent dans ce résultat alors que la démonstration du théorème de Beurling et Nyman était basée sur la question de l'existence des zéros de partie réelle supérieure à $\frac{1}{2}$.

La démonstration de notre résultat est basée sur la construction de vecteurs indexés par les zéros critiques de la fonction $F$ de la classe de Selberg à laquelle on s'intéresse, vecteurs qui seront orthogonaux à un espace proche de $\mathcal{B}_{F}^{\lambda}$. Un calcul de transformée de Mellin permet de fournir des candidats naturels pour ces vecteurs. Cependant, ces candidats n'étant pas des vecteurs de $L^{2}(0,+\infty)$, nous serons amenés à les modifier de manière à conserver leur propriété d'orthogonalité tout en rendant nos calculs formels licites. Une telle modification se fera par l'application d'opérateurs de $L^{2}(0,+\infty)$.

Nous établirons dans le second paragraphe quelques estimations liées à l'utilisation de la formule de Stirling complexe. Nous rappelerons ensuite quelques propriétés des fonctions de Bessel, puis des opérateurs. Le cinquième paragraphe sera consacrée à l'étude de l'action d'un opérateur dit « de phase » associé à la fonction $\Psi_{F}^{(1)}$.

Nous construirons dans le sixième paragraphe des vecteurs indexés par les zéros de la fonction $F$ et vérifierons leurs propriétés d'orthogonalité.

Nous démontrerons le théorème 2 dans la dernière partie. Pour cela, nous minorerons la distance $D(\lambda)$ par la norme de la projection orthogonale d'un vecteur proche de $\chi$ sur le sous-espace vectoriel engendré par les vecteurs que l'on viendra de construire.

Nous supposons désormais que $F$ est une fonction de la classe de Selberg telle que $\Psi_{F}^{(1)} \in L^{2}(0,+\infty)$.

TOME $134-2006-\mathrm{N}^{\mathrm{O}} 3$ 


\section{Quelques conséquences de la formule de Stirling complexe}

Par soucis de clarté, nous regroupons dans ce paragraphe quelques estimations techniques qui proviennent de la formule de Stirling complexe. Nous utiliserons la proposition suivante dont on trouvera une démonstration dans [5] (formule (19), (VII.2.3)).

Proposition 2.1. - Il existe des constantes $c_{\nu}=c_{\nu}(a)$ telles que, pour tout $M \in \mathbb{N}$,

(1) $\log \Gamma(s+a)=\left(s+a-\frac{1}{2}\right) \log s-s+\frac{1}{2} \log 2 \pi+\sum_{\nu=1}^{M} c_{\nu} s^{-\nu}+O\left(\frac{1}{|s|^{M+1}}\right)$

uniformément pour $|\arg (s)| \leq \pi-\varepsilon$ avec $\varepsilon>0$ fixé et a dans un compact de $\mathbb{C}$, $|s|$ tendant vers l'infini.

\section{1. Étude d'une fonction liée à l'équation fonctionnelle de $\boldsymbol{F}$}

Notations 2. - Si $\omega, Q$ et $\Delta(s)=\prod_{j=1}^{r} \Gamma\left(\lambda_{j} s+\mu_{j}\right)$ sont les données de $F$ apparaissant dans l'équation fonctionnelle qu'elle vérifie, on pose

$$
U_{F}(s)=\bar{\omega} Q^{2 s-1} \frac{\Delta(s)}{\bar{\Delta}(1-s)} \cdot \frac{s}{1-s} .
$$

Notations 3. - Pour $a>0$ et $\nu \geq 0$, on pose

$$
f_{a, \nu}(s)=\frac{1}{2}\left(\frac{2}{a}\right)^{s-\nu} \frac{\Gamma\left(\frac{1}{2} s\right)}{\Gamma\left(\nu-\frac{1}{2} s+1\right)} .
$$

Nous allons relier la fonction $U_{F}$ à la fonction $f_{a, \nu}$.

Proposition 2.2. - Si on pose pour $k=0$ ou $k=1$

$$
\nu_{k}=2 \operatorname{Re} \mu+\frac{d}{2}+k, \beta_{k}=2 k-1+2 \mu, \quad a=d\left(Q^{-2} \prod_{j=1}^{r} \lambda_{j}^{-2 \lambda_{j}}\right)^{1 / d},
$$

alors il existe des constantes $c_{1, k}$ et $c_{2, k}$, dépendant uniquement des données de $F$ et de $k$ telles que, lorsque $|s|$ tend vers l'infini, on ait uniformément par rapport à s tel que $\varepsilon<|\arg s|<\pi-\varepsilon$,

$$
\begin{aligned}
\frac{U_{F}(s)}{s^{2-k}}=c_{1, k} f_{a, \nu_{k}}\left(d s+\beta_{k}\right)+c_{2, k} & s^{-1} f_{a, \nu_{k}}\left(d s+\beta_{k}\right) \\
& +O\left(\left|f_{a, \nu_{k}}\left(d s+\beta_{k}\right)\right| \cdot|s|^{-2}\right) .
\end{aligned}
$$

Démonstration. - Dans toute la démonstration, les constantes $k_{i}$ seront des constantes convenables qui ne dépendront que des données de $F$.

BULletin DE LA SOCiÉtÉ MATHÉmATiQUe DE FRANCE 
En utilisant la formule (1), on a pour $\varepsilon \leq|\arg (s)| \leq \pi-\varepsilon$, si $|s|$ tend vers l'infini,

$$
\begin{aligned}
\log \left(\frac{\Delta(s)}{\bar{\Delta}(1-s)}\right)= & \sum_{j=1}^{r}\left(\log \Gamma\left(\lambda_{j} s+\mu_{j}\right)-\log \Gamma\left(-\lambda_{j} s+\lambda_{j}+\bar{\mu}_{j}\right)\right) \\
= & \sum_{j=1}^{r}\left\{\left(\lambda_{j} s+\mu_{j}-\frac{1}{2}\right) \log \left(\lambda_{j} s\right)-\lambda_{j} s\right. \\
\left.-\left(-\lambda_{j} s+\lambda_{j}+\bar{\mu}_{j}-\frac{1}{2}\right) \log \left(-\lambda_{j} s\right)-\lambda_{j} s\right\} & +k_{1} s^{-1}+O\left(s^{-2}\right) \\
= & \frac{1}{2} d s(\log s+\log (-s))+s\left(\sum_{j=1}^{r} 2 \lambda_{j} \log \lambda_{j}-d\right) \\
\quad+\sum_{j=1}^{r}\left(\mu_{j}-\frac{1}{2}\right) \log s-\left(\sum_{j=1}^{r}\left(\bar{\mu}_{j}-\frac{1}{2}\right)+\frac{1}{2} d\right) \log (-s) & +k_{2}+k_{3} s^{-1}+O\left(s^{-2}\right) .
\end{aligned}
$$

On a donc

(4) $\log \frac{U_{F}(s)}{s^{k}}=\frac{1}{2} d s(\log s+\log (-s))+s\left(2 \log Q+2 \sum_{j=1}^{r} \lambda_{j} \log \lambda_{j}-d\right)$

$$
+(1+\mu-k) \log s-\left(\bar{\mu}+\frac{1}{2} d+1\right) \log (-s)+k_{4}+k_{5} s^{-1}+O\left(s^{-2}\right),
$$

uniformément pour $s$ tel que $\varepsilon<|\arg s|<\pi-\varepsilon$ avec $\varepsilon>0,|s|$ tendant vers l'infini.

D'autre part, pour $\alpha>0, a>0, \nu \in \mathbb{R}$ et $\beta \in \mathbb{C}$, on a

$$
\begin{aligned}
\log \left(f_{a, \nu}(\alpha s+\beta)\right)=\alpha s(\log 2-\log a) & +\log \Gamma\left(\frac{1}{2}(\alpha s+\beta)\right) \\
& -\log \Gamma\left(\nu-\frac{1}{2}(\alpha s+\beta)+1\right)+\ell_{1} .
\end{aligned}
$$

En utilisant la formule de Stirling (1), on obtient

$$
\begin{aligned}
& \log \left(f_{a, \nu}(\alpha s+\beta)\right)=\frac{1}{2} \alpha s(\log s+\log (-s))+\alpha(\ln \alpha-\ln a-1) s \\
& \quad+\frac{1}{2}(\beta-1) \log s+\left(\frac{1}{2}(\beta-1)-\nu\right) \log (-s)+\ell_{2}+\ell_{3} s^{-1}+O\left(s^{-2}\right)
\end{aligned}
$$

uniformément pour $s$ tel que $\varepsilon<|\arg s|<\pi-\varepsilon$ avec $\varepsilon>0,|s|$ tendant vers l'infini, où les constantes $\ell_{i}$ dépendent de $\alpha, a, \nu$ et $\beta$.

En comparant (4) et (5), on obtient

$$
\log \frac{U_{F}(s)}{s^{2-k}}=\log f_{a, \nu_{k}}\left(d s+\beta_{k}\right)+k_{6}+k_{7} s^{-1}+O\left(s^{-2}\right) .
$$

TOME $134-2006-\mathrm{N}^{\mathrm{O}} 3$ 
Il existe donc des constantes $c_{1, k}$ et $c_{2, k}$, dépendant uniquement des données de $F$ et de $k$ telles que, lorsque $|s|$ tend vers l'infini, on ait uniformément par rapport à $s$ tel que $\varepsilon<|\arg s|<\pi-\varepsilon$,

$$
\begin{aligned}
\frac{U_{F}(s)}{s^{2-k}}=c_{1, k} f_{a, \nu_{k}}\left(d s+\beta_{k}\right) & +c_{2, k} s^{-1} f_{a, \nu_{k}}\left(d s+\beta_{k}\right) \\
& +O\left(\left|f_{a, \nu_{k}}\left(d s+\beta_{k}\right)\right| \cdot|s|^{-2}\right) .
\end{aligned}
$$

Proposition 2.3. - On a

$$
\left|U_{F}(\sigma+i \tau)\right| \asymp|\tau|^{d\left(\sigma-\frac{1}{2}\right)}
$$

uniformément pour $\sigma \in\left[\sigma_{1}, \sigma_{2}\right]$ et $|\tau|$ tendant vers l'infini. Pour $a>0$ et $\nu \in \mathbb{R}$, si $|\tau|$ tend vers l'infini, on a

$$
\left|f_{a, \nu}(\sigma+i \tau)\right| \asymp|\tau|^{\sigma-1-\nu}
$$

uniformément pour $\sigma \in\left[\sigma_{1}, \sigma_{2}\right]$.

Démonstration. - Ces estimations se déduisent facilement de (4) et (5).

\subsection{Estimation d'un quotient de fonctions $\Gamma$ en certains points.}

Proposition 2.4. - Pour $\nu \in \mathbb{R}, \operatorname{Re} z=N+\frac{1}{2}$ avec $N \in \mathbb{N}$ et $N \geq|\nu|+\frac{1}{2}$, on $a$

$$
\frac{\Gamma(1-z)}{\Gamma(\nu+z)} \ll \frac{\mathrm{e}^{2 N}}{|z|^{N}|\nu+z|^{N+\nu}}
$$

Démonstration. — En utilisant la formule des compléments, on obtient :

$$
\frac{\Gamma(1-z)}{\Gamma(\nu+z)}=\frac{\pi / \sin (\pi z)}{\Gamma(\nu+z) \Gamma(z)}
$$

On a pour $z=N+\frac{1}{2}+i t$,

$$
\left|\frac{\pi}{\sin (\pi z)}\right|=\left|\frac{2 i \pi}{\mathrm{e}^{i \pi\left(N+\frac{1}{2}\right)} \mathrm{e}^{-\pi t}-\mathrm{e}^{-i \pi\left(N+\frac{1}{2}\right)} \mathrm{e}^{\pi t}}\right|=\frac{2 \pi}{\mathrm{e}^{-\pi|t|}+\mathrm{e}^{\pi|t|}} .
$$

Évaluons à présent le produit de fonctions $\Gamma$. D'après (1), on a uniformément pour $\operatorname{Re} z \geq|\nu|+1$

$$
\begin{gathered}
\log |\Gamma(\nu+z) \Gamma(z)|=\left(\operatorname{Re} z-\frac{1}{2}\right) \log |z|+\left(\nu+\operatorname{Re} z-\frac{1}{2}\right) \log |\nu+z|-|\operatorname{Im} z| \pi \\
+\operatorname{Im} z\left(\arctan \frac{\operatorname{Re} z}{\operatorname{Im} z}+\arctan \frac{\operatorname{Re} z+\nu}{\operatorname{Im} z}\right) \\
-2 \operatorname{Re} z-\nu+\log 2 \pi+O\left(\frac{1}{|z|}\right) \\
\geq\left(\operatorname{Re} z-\frac{1}{2}\right) \log |z|+\left(\nu+\operatorname{Re} z-\frac{1}{2}\right) \log |\nu+z| \\
-|\operatorname{Im} z| \pi-2 \operatorname{Re} z-\nu+\log 2 \pi+O\left(\frac{1}{|z|}\right) .
\end{gathered}
$$

BULLETIN DE LA SOCiÉTÉ MATHÉMATIQUe DE FRANCE 
On obtient pour $N \geq|\nu|+\frac{1}{2}$ et $4 z=N+\frac{1}{2}+i t$,

$$
\begin{aligned}
\log |\Gamma(\nu+z) \Gamma(z)| \geq N \log |z| & +(\nu+N) \log |\nu+z|-|t| \pi \\
& -2 N-1-\nu+\log 2 \pi+O\left(\frac{1}{|z|}\right) .
\end{aligned}
$$

On a donc

$$
\frac{\Gamma(1-z)}{\Gamma(\nu+z)} \ll|z|^{-N}|\nu+z|^{-\nu-N} \mathrm{e}^{2 N}
$$

On a donc bien la majoration annoncée.

\section{Fonctions de Bessel}

Les fonctions de Bessel sont étudiées dans le livre de Watson [12] qui est notre principale référence dans ce paragraphe. Nous en rappelons ici quelques propriétés et nous en donnons une représentation intégrale (proposition 3.1) qui ne se trouve pas dans [12].

Notations 4. - Si $\sigma \in \mathbb{R}$, désormais $\int_{(\sigma)}$ désignera l'intégrale $\int_{\sigma-i \infty}^{\sigma+i \infty}$.

DÉfinition 3. - Soit $\nu$ un réel. On définit la fonction de Bessel $J_{\nu}$ comme suit (formule (8), § 3.1 de [12]) :

$$
\forall x \in \mathbb{R}, \quad J_{\nu}(x)=\sum_{m=0}^{+\infty} \frac{(-1)^{m}\left(\frac{1}{2} x\right)^{\nu+2 m}}{m ! \Gamma(\nu+m+1)} .
$$

Cette série converge absolument sur $\mathbb{R}^{+*}$ et la convergence est uniforme pour $x$ dans un compact donné de $\mathbb{R}^{+*}$ et $\nu$ dans un compact de $\mathbb{R}$ ( $\$ 3.13$ de [12]).

Soit $\nu \in \mathbb{R}$. Lorsque $x$ tend vers l'infini, la fonction $J_{\nu}$ vérifie

$$
J_{\nu}(x)=O\left(\frac{1}{\sqrt{x}}\right) .
$$

Nous allons exprimer la fonction $x^{-\nu} J_{\nu}$ comme une intégrale proche d'une transformée de Mellin inverse. Nous savons déjà (formule (7), §6.5 de [12]) que si $\nu \geq 0$, alors pour tout $s \in \mathbb{C}$ tel que Re $s \in] 0, \nu+1[$, on a

$$
\mathcal{M}\left(x^{-\nu} J_{\nu}(x)\right)(s)=\frac{2^{s-\nu-1} \Gamma\left(\frac{1}{2} s\right)}{\Gamma\left(\nu-\frac{1}{2} s+1\right)} .
$$

Nous allons étendre ce résultat.

DÉfinition 4. - Soient $T>0, \Lambda$ et $c$ des réels tels que $c \leq \Lambda$. On considère le contour

$$
C(T, c, \Lambda):=] c-i \infty ; c-i T ; \Lambda-i T ; \Lambda+i T ; c+i T ; c+i \infty[.
$$

Énonçons à présent quelques lemmes préliminaires.

TOME $134-2006-\mathrm{N}^{\mathrm{O}} 3$ 
LEMME 1. - Soit $f$ une fonction méromorphe sur $\mathbb{C}$ vérifiant :

1) $T_{0}:=\sup \{|\operatorname{Im} \rho| ; \rho$ pôle de $f\}<+\infty$;

2) $\Lambda_{0}:=\sup \{\operatorname{Re} \rho ; \rho$ pôle de $f\}<+\infty$;

3) il existe $a>0$ et $b \in \mathbb{R}$ tels que, pour tout $\sigma_{1}<\sigma_{2}$, on ait

$$
|f(s)| \ll|\tau|^{a \sigma-b-1}
$$

uniformément pour $\sigma \in\left[\sigma_{1}, \sigma_{2}\right]$ et $|\tau|$ tendant vers l'infini.

Si T, c et $\Lambda$ vérifient les conditions

$$
T>T_{0}, \quad c<\frac{b}{a} \quad \text { et } \quad \Lambda>\Lambda_{0}, \Lambda \geq c
$$

alors l'intégrale

$$
I(x):=\frac{1}{2 i \pi} \int_{C(T, c, \Lambda)} f(s) x^{-s} \mathrm{~d} s
$$

converge pour $x>0$ et sa valeur ne dépend pas des paramètres $T$, $c$ et $\Lambda$ vérifiant (9). De plus, pour tout $\varepsilon>0$, on a lorsque $x$ tend vers l'infini,

$$
I(x)=O_{\varepsilon}\left(x^{-b / a+\varepsilon}\right) .
$$

Démonstration. - Il suffit d'appliquer le théorème des résidus entre deux contours vérifiant les hypothèses (9).

Lemme 2. - Soient $a, b \in \mathbb{R}^{+*}$. Supposons $b \geq 1$. Alors on $a$

$$
\int_{0}^{+\infty} \frac{\mathrm{d} \tau}{\left(a^{2}+\tau^{2}\right)^{b}} \leq \frac{\pi}{2} \frac{a^{1-2 b}}{\sqrt{b}}
$$

Démonstration. - Remarquons dans un premier temps que puisque $a>0$ et $b>\frac{1}{2}$, l'intégrale est bien convergente. On a de plus, en effectuant le changement de variable $\tau^{\prime}=\tau / a$,

$$
\int_{0}^{+\infty} \frac{\mathrm{d} \tau}{\left(a^{2}+\tau^{2}\right)^{b}}=a^{1-2 b} \int_{0}^{+\infty} \frac{\mathrm{d} \tau}{\left(1+\tau^{2}\right)^{b}}
$$

Un calcul avec MAPLE donne

$$
\int_{0}^{+\infty} \frac{\mathrm{d} \tau}{\left(1+\tau^{2}\right)^{b}}=\frac{\sqrt{\pi}}{2} \cdot \frac{\Gamma\left(b-\frac{1}{2}\right)}{\Gamma(b)} .
$$

La fonction $\varphi(b)=\sqrt{b} \Gamma\left(b-\frac{1}{2}\right) / \Gamma(b)$ admet comme dérivée logarithmique $\frac{\varphi^{\prime}(b)}{\varphi(b)}=\frac{1}{2 b}+\sum_{q \in \mathbb{N}}\left(\frac{1}{q+b}-\frac{1}{q+b-\frac{1}{2}}\right) \leq \frac{1}{2 b}+\int_{0}^{+\infty}\left(\frac{1}{t+b}-\frac{1}{t+b-\frac{1}{2}}\right) \mathrm{d} t \leq 0$.

On a donc pour $b \geq 1$,

$$
\int_{0}^{+\infty} \frac{\mathrm{d} \tau}{\left(a^{2}+\tau^{2}\right)^{b}}=a^{1-2 b} \frac{\sqrt{\pi}}{2} \cdot \frac{\Gamma\left(b-\frac{1}{2}\right)}{\Gamma(b)} \leq \frac{\sqrt{\pi}}{2} \cdot \frac{a^{1-2 b}}{\sqrt{b}} \varphi(1)=\frac{\pi}{2} \cdot \frac{a^{1-2 b}}{\sqrt{b}} .
$$

BULletin DE LA SOCIÉtÉ MATHÉMATIQUE DE FRANCE 
Lemme 3. - Soient $a>0$ et $\nu \geq 0$. Si $f_{a, \nu}$ est la fonction définie dans la notation 3 , alors on a uniformément pour $x$ dans un compact de $\mathbb{R}^{+*}$ :

$$
\lim _{N \rightarrow+\infty} \int_{(-2 N+1)} f_{a, \nu}(s) x^{-s} \mathrm{~d} s=0 .
$$

Démonstration. - Posons

$$
K_{N}(x):=\int_{(-2 N+1)} f_{a, \nu}(s) x^{-s} \mathrm{~d} s .
$$

Pour $N \geq|\nu|+1$, on a en posant $z=1-\frac{1}{2} s$ et en utilisant la proposition 2.4 :

$$
\begin{aligned}
K_{N}(x) & =\int_{\left(N+\frac{1}{2}\right)}\left(\frac{2}{a}\right)^{2-2 z-\nu} \frac{\Gamma(1-z)}{\Gamma(\nu+z)} x^{2 z-2} \mathrm{~d} z \\
& =O\left(\frac{1}{x}\left(\frac{e a x}{2}\right)^{2 N} \int_{\left(N+\frac{1}{2}\right)} \frac{|\mathrm{d} z|}{|z|^{N}|\nu+z|^{N+\nu}}\right) .
\end{aligned}
$$

Or si $u, v \geq 1$, on a $1 /(u v) \leq 1 / u+1 / v$. On a donc

Or on a d'après le lemme 2,

$$
K_{N}(x)=O\left(\frac{1}{x}\left(\frac{e a x}{2}\right)^{2 N}\left(\int_{\left(N+\frac{1}{2}\right)} \frac{|\mathrm{d} z|}{|z|^{N}}+\int_{\left(N+\frac{1}{2}\right)} \frac{|\mathrm{d} z|}{|\nu+z|^{N+\nu}}\right)\right) .
$$

$\int_{\left(N+\frac{1}{2}\right)} \frac{|\mathrm{d} z|}{|z|^{N}}=\int_{-\infty}^{+\infty} \frac{\mathrm{d} t}{\left(\left(N+\frac{1}{2}\right)^{2}+t^{2}\right)^{\frac{1}{2} N}} \ll \frac{\left(N+\frac{1}{2}\right)^{1-N}}{\sqrt{N}}$

et

$\int_{\left(N+\frac{1}{2}\right)} \frac{|\mathrm{d} z|}{|\nu+z|^{N+\nu}}=\int_{-\infty}^{+\infty} \frac{\mathrm{d} t}{\left(\left(N+\nu+\frac{1}{2}\right)^{2}+t^{2}\right)^{\frac{1}{2}(N+\nu)}} \ll \frac{\left(N+\nu+\frac{1}{2}\right)^{1-N-\nu}}{\sqrt{N+\nu}}$. Si $N \geq 2|\nu|+1$, on a donc

$$
K_{N}(x)=O\left(\frac{1}{x} \sqrt{N}\left(\frac{e a x}{2}\right)^{2 N}\left(N^{-N}+\left(\frac{N}{2}\right)^{-\frac{1}{2} N}\right)\right) .
$$

Il est donc clair que l'intégrale $K_{N}(x):=\int_{(-2 N+1)} f(s) x^{-s} \mathrm{~d} s$ tend vers 0 lorsque $N$ tend vers l'infini uniformément pour $x$ dans un compact de $\mathbb{R}^{+*}$.

Proposition 3.1. - Si $\nu \in \mathbb{R}$ et si

$$
T \geq 1, \quad c<\nu \quad \text { et } \Lambda>0, \Lambda \geq c,
$$

alors l'intégrale

$$
\int_{C(T, c, \Lambda)} f_{a, \nu}(s) x^{-s} \mathrm{~d} s
$$

est convergente et sa valeur ne dépend pas des paramètres $T$, c et $\Lambda$ vérifiant les conditions (10). De plus, on a

$$
x^{-\nu} J_{\nu}(a x)=\frac{1}{2 i \pi} \int_{C(T, c, \Lambda)} f_{a, \nu}(s) x^{-s} \mathrm{~d} s .
$$

TOME $134-2006-\mathrm{N}^{\mathrm{O}} 3$ 
Démonstration. - Afin d'alléger les notations, on écrira $f$ pour $f_{a, \nu}$ dans cette démonstration.

Les pôles de la fonction $f_{a, \nu}(\alpha s+\beta)$ sont les points $-(2 n+\beta) / \alpha, n \in \mathbb{N}$. Le lemme 1 et l'estimation (7) permettent de montrer la première partie de la proposition.

Montrons maintenant que l'intégrale

$$
g(x):=\frac{1}{2 i \pi} \int_{C(T, c, \Lambda)} f(s) x^{-s} \mathrm{~d} s
$$

est bien égale à $x^{-\nu} J_{\nu}(a x)$. Soit $N>\frac{1}{2}(1-\nu)$ un entier naturel positif. D'après le lemme 1 , comme $-2 N+1<\nu$, on a

$$
g(x)=\frac{1}{2 i \pi} \int_{C(T,-2 N+1, \Lambda)} f(s) x^{-s} \mathrm{~d} s .
$$

Appliquons maintenant le théorème des résidus à la fonction $f(s) x^{-s}$ sur le rectangle $R:=[-2 N+1-i T, \Lambda-i T, \Lambda+i T,-2 N+1+i T]$. On obtient

$$
g(x)=\frac{1}{2 i \pi} \int_{(-2 N+1)} f(s) x^{-s} \mathrm{~d} s+\sum_{m=0}^{N-1} p_{m} x^{2 m},
$$

où $p_{m}=\operatorname{Res}(f(s),-2 m)=(2 / a)^{-2 m-\nu}(-1)^{m} /(m ! \Gamma(\nu+m+1))$.

D'après le lemme 3 , l'intégrale $K_{N}(x):=\int_{(-2 N+1)} f(s) x^{-s} \mathrm{~d} s$ tend vers 0 lorsque $N$ tend vers l'infini uniformément pour $x$ dans un compact de $\mathbb{R}^{+*}$. On a donc, en faisant tendre $N$ vers l'infini dans (11),

$$
g(x)=\sum_{m \geq 0} p_{m} x^{2 m}=x^{-\nu} J_{\nu}(a x) .
$$

\section{Opérateurs invariants}

4.1. Rappels. - Afin de donner des versions rigoureuses de calculs formels, nous travaillerons avec des opérateurs, linéaires et semi-linéaires, de l'espace $L^{2}(0,+\infty)$.

DÉFInItion 5. - Soit $\theta$ un réel stictement positif. On définit l'opérateur de dilatation-contraction $D_{\theta}$ par

$$
D_{\theta} f(t)=\frac{1}{\sqrt{\theta}} f\left(\frac{t}{\theta}\right)
$$

pour toute fonction $f$ de $L^{2}(0,+\infty)$ et tout réel $t>0$. L'opérateur $D_{\theta}$ sera appelé opérateur de dilatation si $\theta>1$ et de contraction sinon.

Remarquons que si $L^{2}(0,1)$ désigne l'ensemble des fonctions de $L^{2}(0,+\infty)$ identiquement nulles sur $[1,+\infty[$, ce que nous supposerons implicitement par la suite, les contractions laissent $L^{2}(0,1)$ stable. 
DÉfinition 6. - Un opérateur $T$ sur un sous-espace de $L^{2}(0,+\infty)$ sera dit invariant s'il commute avec les dilatations-contractions, i.e. si pour tout $\theta>0$, on a $D_{\theta} T=T D_{\theta}$.

Donnons à présent quelques exemples d'opérateurs linéaires et semi-linéaires qui nous seront utiles par la suite.

EXEMPLE 1. - On définit :

- l'opérateur de Hardy $H$ (qui est un opérateur invariant) par

$$
H: f(t) \longmapsto \frac{1}{t} \int_{0}^{t} f(u) \mathrm{d} u
$$

- le projecteur orthogonal $Q_{\lambda}$ de $L^{2}(0,+\infty)$ sur $L^{2}(\lambda,+\infty)$ pour tout $\lambda>0$;

- l'opérateur d'inversion $J$ ( $J$ n'est pas linéaire mais semi-linéaire), par

$$
J: f(t) \longmapsto \frac{1}{t} \overline{f\left(\frac{1}{t}\right)} .
$$

On remarquera que l'image de $L^{2}(0,1)$ par l'opérateur $J$ est égale à $L^{2}(1,+\infty)$.

La transformation de Mellin-Plancherel

$$
\mathcal{M}: f(t) \longmapsto \mathcal{M} f(s)=\int_{0}^{+\infty} f(u) u^{s-1} \mathrm{~d} u
$$

établit une isométrie entre $L^{2}(0,+\infty)$ et $L^{2}\left(s=\frac{1}{2}+i \tau, \frac{1}{2 \pi} \mathrm{d} \tau\right)$ dont l'inverse est

$$
\mathcal{M}^{-1}: F(s) \longmapsto \int_{s=\frac{1}{2}+i \tau} F(s) t^{-s} \frac{\mathrm{d} \tau}{2 \pi} .
$$

Rappelons qu'ici,

- $\int_{0}^{+\infty} f(u) u^{s-1} \mathrm{~d} u$ désigne la limite dans $L^{2}(0,+\infty)$, lorsque $T$ tend vers l'infini, de $\int_{1 / T}^{T} f(u) u^{s-1} \mathrm{~d} u$;

- $\int_{s=\frac{1}{2}+i \tau} F(s) t^{-s} \mathrm{~d} \tau / 2 \pi$ désigne la limite dans $L^{2}(\mathbb{R})$, lorsque $T$ tend vers l'infini, de $\int_{-T}^{T} F\left(\frac{1}{2}+i \tau\right) t^{-\frac{1}{2}-i \tau} \frac{1}{2 \pi} \mathrm{d} \tau$.

On notera $\|$.$\| les normes dans L^{2}(0,+\infty)$ et $L^{2}\left(\frac{1}{2}+i \tau, \frac{1}{2 \pi} \mathrm{d} \tau\right)$.

Rappelons (voir par exemple [7]) que si $T(s)$ est une fonction Lebesguemesurable et essentiellement bornée sur $\operatorname{Re} s=\frac{1}{2}$, alors la transformation $F(s) \mapsto T(s) F(s)$ définit via la transformation de Mellin inverse un opérateur borné invariant que l'on notera aussi $T$ sur $L^{2}(0,+\infty)$ et que tout opérateur borné invariant peut être obtenu de cette manière. La classe d'équivalence pour l'égalité presque partout de la fonction $T(s)$ est appelée le symbole de l'opérateur $T$. Nous noterons également $T(s)$ toute extension méromorphe de la fonction $T(s)$ initialement définie sur la droite critique. Remarquons que si $|T(s)|=1$ presque partout sur la droite critique, alors l'opérateur $T$ est unitaire.

TOME $134-2006-\mathrm{N}^{\mathrm{O}} 3$ 
Exemple 2. - Le symbole associé à l'opérateur de Hardy $H$ est la fonction $H(s)=1 /(1-s)$. L'opérateur $1-H$ admet $s /(1-s)$ pour symbole et est donc unitaire.

Nous introduisons ici la notion d'opérateur de phase, définie par J.-F. Burnol dans [7] :

Proposition-Définition 1. - Soit $f \in L^{2}(0,+\infty)$ une fonction dont la transformée de Mellin est presque partout non nulle sur $\operatorname{Re} s=\frac{1}{2}$. La fonction de phase associée à $f$, définie sur $\operatorname{Re} s=\frac{1}{2}$ par

$$
U_{f}(s):=\frac{\overline{\mathcal{M} f(s)}}{\mathcal{M} f(s)}
$$

est presque partout définie et de module 1. L'opérateur $U_{f}$ qu'on lui associe est appelé opérateur de phase associé à la fonction $f$; il est invariant, unitaire et vérifie $U_{f}(f)=J(f)$. En particulier, si $f \in L^{2}(0,1)$, alors $U_{f} f \in L^{2}(1,+\infty)$.

Comme annoncé précédemment, on notera $U_{f}(s)$ tout prolongement méromorphe de la fonction $U_{f}(s)$ définie originellement sur $\operatorname{Re} s=\frac{1}{2}$.

La proposition suivante nous permet de caractériser l'action de certains opérateurs à l'aide de leur action sur la fonction indicatrice $\chi$.

Proposition 4.1. - Soit $U: L^{2}(0,+\infty) \rightarrow L^{2}(0,+\infty)$ un opérateur borné invariant. On suppose que la fonction $\varphi:=U \chi$ est de classe $C^{1}$ sur $\mathbb{R}^{+*}$. On suppose de plus que la fonction $\Phi$ définie sur $\mathbb{R}^{+*}$ par

$$
\Phi(x):=x \varphi^{\prime}(x)
$$

appartient à $L^{2}(0,+\infty)$. Alors pour toute fonction $f$ de $L^{2}(0,+\infty)$, la fonction Uf est définie par la limite suivante dans $L^{2}(0,+\infty)$ (cette limite existe bien) :

$$
U f(t):=\lim _{T \rightarrow+\infty} \int_{1 / T}^{T} f(v) \frac{\mathrm{d}}{\mathrm{d} v} U \chi\left(\frac{t}{v}\right) \mathrm{d} v .
$$

Démonstration. - Soit $\theta>0$. Pour $T \geq \max (\theta, 1 / \theta)$, on a

$$
\begin{aligned}
g_{T}(t) & :=\int_{1 / T}^{T} D_{\theta} \chi(v) \frac{\mathrm{d}}{\mathrm{d} v} \varphi\left(\frac{t}{v}\right) \mathrm{d} v \\
& =\theta^{-\frac{1}{2}} \int_{1 / T}^{\theta} \frac{\mathrm{d}}{\mathrm{d} v} \varphi\left(\frac{t}{v}\right) \mathrm{d} v=\theta^{-\frac{1}{2}}\left(\varphi\left(\frac{t}{\theta}\right)-\varphi(T t)\right) .
\end{aligned}
$$

Or $\int_{0}^{+\infty}|\varphi(T t)|^{2} \mathrm{~d} t=T^{-1}\|\varphi\|^{2}$, donc la limite de la fonction $g_{T}$ dans $L^{2}(0,+\infty)$ lorsque $T$ tend vers $+\infty$ est la fonction $D_{\theta} \varphi=U D_{\theta} \chi$ (car $U$ est invariant). Par linéarité, on en déduit la propriété recherchée pour les fonctions $f$ en escalier. 
Reste à démontrer la propriété pour toutes les fonctions de $L^{2}(0,+\infty)$. Soit $T>0$. Considérons l'opérateur $K_{T}$ défini sur $L^{2}(0,+\infty)$ par

$$
K_{T} f(x)=\int_{1 / T}^{T} f(v) \frac{\mathrm{d}}{\mathrm{d} v} \varphi\left(\frac{x}{v}\right) \mathrm{d} v .
$$

Si $f \in L^{2}(0,+\infty)$, alors l'intégrale définissant $K_{T} f$ est absolument convergente. Nous allons montrer que $K_{T}$ est un opérateur linéaire borné de $L^{2}(0,+\infty)$ à valeurs dans $L^{2}(0,+\infty)$. Pour $f \in L^{2}(0,+\infty)$, en posant $u=1 / v$, on a

$$
K_{T} f(x)=-\int_{1 / T}^{T} \frac{1}{u} f\left(\frac{1}{u}\right) \Phi(x u) \mathrm{d} u,
$$

ce qui montre que $K_{T} f$ est continue sur $\mathbb{R}^{+*}$. D'autre part,

$$
\begin{aligned}
\left|K_{T} f(x)\right|^{2} & \leq\left(\int_{1 / T}^{T} \frac{1}{u^{2}}\left|f\left(\frac{1}{u}\right)\right|^{2} \mathrm{~d} u\right)\left(\int_{1 / T}^{T}|\Phi(x u)|^{2} \mathrm{~d} u\right) \\
& \leq\|f\|^{2}\left(\int_{1 / T}^{T}|\Phi(x u)|^{2} \mathrm{~d} u\right) .
\end{aligned}
$$

Or

$$
\begin{aligned}
\int_{0}^{+\infty} \int_{1 / T}^{T}|\Phi(x u)|^{2} \mathrm{~d} u \mathrm{~d} x & =\int_{1 / T}^{T} \int_{0}^{+\infty}|\Phi(x u)|^{2} \mathrm{~d} x \mathrm{~d} u \\
& =\|\Phi\|^{2} \int_{1 / T}^{T} \frac{\mathrm{d} u}{u}=2\|\Phi\|^{2} \log T
\end{aligned}
$$

la fonction $K_{T} f$ est donc une fonction de $L^{2}(0,+\infty)$ et

$$
\left\|K_{T} f\right\| \leq \sqrt{2 \log T}\|\Phi\| \cdot\|f\| .
$$

L'opérateur $K_{T}$ est donc continu à valeurs dans $L^{2}(0,+\infty)$.

L'opérateur $U_{T}$ défini par $U_{T} f=U\left(f_{[1 / T, T]}\right)$ où $f_{[1 / T, T]}$ est la restriction de la fonction $f$ à l'intervalle $[1 / T, T]$, est continu à valeurs dans $L^{2}(0,+\infty)$ et coïncide avec $K_{T}$ sur l'ensemble dense des fonctions en escalier, donc $K_{T}=U_{T}$.

Si $f \in L^{2}(0,+\infty)$, par continuité de l'opérateur $U, U f$ est la limite quand $T$ tend vers l'infini de $U\left(f_{[1 / T, T]}\right)=K_{T} f$. On a donc bien la propriété recherchée.

\section{5. Étude de l'opérateur de phase associé à $\Psi_{F}^{(1)}$}

Rappelons (voir [9]) que pour $\frac{1}{2}<\sigma<1$,

$$
\mathcal{M} \Psi_{F}^{(1)}(s)=-\frac{F(s)}{s} .
$$

TOME $134-2006-\mathrm{N}^{\mathrm{O}} 3$ 
La fonction $F$ appartient à la classe de Selberg donc la fonction $\mathcal{M} \Psi_{F}^{(1)}$ se prolonge méromorphiquement à $\mathbb{C}$ en une fonction presque partout non nulle sur $\operatorname{Re} s=\frac{1}{2}$.

On peut donc définir l'opérateur de phase $U_{F}$ de la fonction $\Psi_{F}^{(1)}$. Son symbole associé $U_{F}(s)$ qui est égal à $s \overline{F(s)} /(\bar{s} F(s))$ sur la droite critique se prolonge méromorphiquement à $\mathbb{C}$ en la fonction

$$
U_{F}(s)=\frac{\bar{F}(1-s)}{F(s)} \cdot \frac{s}{1-s}=\bar{\omega} Q^{2 s-1} \frac{\Delta(s)}{\bar{\Delta}(1-s)} \cdot \frac{s}{1-s} .
$$

On retrouve la fonction $U_{F}$ introduite en (2).

Nous allons décrire ici l'action de l'opérateur $U_{F}$ sur une fonction de $L^{2}(0,+\infty)$. La proposition 4.1 nous montre que pour étudier l'action de $U_{F}$, il nous faut d'abord étudier les fonctions $\varphi(x)=U_{F} \chi(x)$ et $\Phi(x)=x \varphi^{\prime}(x)$.

LEMME 4. - Les fonctions $\varphi(x)=U_{F} \chi(x)$ et $\Phi(x)=x \varphi^{\prime}(x)$ sont bien définies sur $\mathbb{R}^{+}$et pour tout $\varepsilon>0$, au voisinage de 0 , on a

$$
\varphi(x)=O\left(x^{-\varepsilon}\right) \quad \text { et } \quad \Phi(x)=O\left(x^{-\varepsilon}\right) .
$$

Démonstration. - Pour étudier les fonctions $\varphi$ et $\Phi$, nous considérons les transformées de Mellin inverses des fonctions $U_{F}(s) / s$ et $U_{F}(s)$.

Les pôles de la fonction $U_{F}(s)$ sont les points 1 et $-\left(n+\mu_{j}\right) / \lambda_{j}$, avec $n \in \mathbb{N}$, $j=1, \ldots, r$. On a

$$
\varphi(x)=\frac{1}{2 \pi} \int_{s=\frac{1}{2}+i \tau} \frac{U_{F}(s)}{s} x^{-s} \mathrm{~d} \tau,
$$

donc d'après le lemme 1 , l'estimation (6) et une application du théorème des résidus, on a pour tous $T>\max _{1 \leq j \leq r}\left|\operatorname{Im} \mu_{j}\right| / \lambda_{j}, 0<\Lambda<1$ et $c<\frac{1}{2}$,

$$
\varphi(x)=\frac{1}{2 \pi} \int_{C(T, c, \Lambda)} \frac{U_{F}(s)}{s} x^{-s} \mathrm{~d} \tau .
$$

En choisissant $c<\frac{1}{2}-\frac{1}{d}$, on montre que la fonction $\varphi$ est de classe $C^{1}$ et la fonction $\Phi$ est définie par

$$
\Phi(x)=-\frac{1}{2 \pi} \int_{C\left(T, c^{\prime}, \Lambda\right)} U_{F}(s) x^{-s} \mathrm{~d} \tau,
$$

pour tous $T>\max _{1 \leq j \leq r}\left|\operatorname{Im} \mu_{j}\right| / \lambda_{j}, 0<\Lambda<1$ et $c^{\prime}<\frac{1}{2}-\frac{1}{d}$. En choisissant $c<0, c^{\prime}<0$ et $\Lambda$ petit dans les écritures intégrales de $\varphi$ et $\Phi$, on établit que pour tout $\varepsilon>0$, au voisinage de 0 , on a $\varphi(x)=O\left(x^{-\varepsilon}\right)$ et $\Phi(x)=O\left(x^{-\varepsilon}\right)$.

Nous allons à présent donner une estimation des fonctions $\varphi$ et $\Phi$ en $+\infty$. L'un de nos objectifs est de montrer que la fonction $\Phi$ appartient à l'espace $L^{2}(0,+\infty)$ afin de pouvoir appliquer la proposition 4.1.

BULLETIN DE LA SOCIÉtÉ MATHÉMATIQUE DE FRANCE 
Proposition 5.1. - On a

$$
\varphi(x)=O\left(x^{-\frac{1}{2}\left(1+\frac{1}{d}\right)}\right) \quad \text { et } \quad \Phi(x)=O\left(x^{-\frac{1}{2}\left(1+\frac{1}{d}\right)}\right) .
$$

Démonstration. — D'après le lemme 1 , si

$$
T>T_{0}:=\max _{j=1, \ldots, r} \frac{\left|\operatorname{Im} \mu_{j}\right|}{\lambda_{j}}, \quad c_{k}<\frac{1}{2}-\frac{k}{d} \quad \text { et } \quad \Lambda>1,
$$

alors l'intégrale

$$
\int_{C\left(T, c_{k}, \Lambda\right)} U_{F}(s) s^{k-1} x^{-s} \mathrm{~d} s
$$

est convergente et sa valeur ne dépend pas des paramètres $T, c_{k}$ et $\Lambda$ vérifiant les conditions (13). Pour $k=0$ ou $k=1$, on définit donc la fonction $I_{k}$ par

$$
I_{k}(x):=\frac{1}{2 i \pi} \int_{C_{1, k}} U_{F}(s) s^{k-1} x^{-s} \mathrm{~d} s,
$$

où $C_{1, k}=C\left(T, c_{k}, \Lambda\right)$ avec $T, c_{k}$ et $\Lambda$ vérifiant les conditions (13). Avec ces notations, on a

$$
\varphi(x)=I_{0}(x)-\frac{1}{x} \operatorname{Res}\left(\frac{U_{F}(s)}{s}, 1\right) \quad \text { et } \quad \Phi(x)=-I_{1}(x)+\frac{1}{x} \operatorname{Res}\left(U_{F}(s), 1\right) .
$$

Nous allons utiliser la fonction $f_{a, \nu}$, définie à la notation 3 , afin de relier les fonctions $\varphi$ et $\Phi$ aux fonctions de Bessel $J_{\nu}$. Reprenons les notations de la proposition 2.2 et posons

$$
r_{k}(s):=\frac{U_{F}(s)}{s^{1-k}}-s c_{1, k} f_{a, \nu_{k}}\left(d s+\beta_{k}\right)-c_{2, k} f_{a, \nu_{k}}\left(d s+\beta_{k}\right) .
$$

En utilisant la proposition 2.2 et l'estimation (7), on a

$$
r_{k}(s)=O\left(|\tau|^{d\left(\sigma-\frac{1}{2}\right)+k-3}\right) .
$$

Les pôles de $r_{k}$ sont les points 0,1 , et les points de la forme $-\left(2 \ell+\beta_{k}\right) / d$ et $-\left(2 \ell+\mu_{j}\right) / \lambda_{j}$ où $\ell \in \mathbb{N}$ et $j \in[1, r]$. En utilisant le lemme 1 , on obtient donc que pour $C_{2, k}=C\left(T, c_{k}, \Lambda\right)$ avec

$$
\left\{\begin{array}{l}
c_{k}<\frac{1}{2}+\frac{2-k}{d}, \\
T>\max \left(\max _{1 \leq j \leq r} \frac{\left|\operatorname{Im} \mu_{j}\right|}{\lambda_{j}}, \frac{2|\operatorname{Im} \mu|}{d}\right), \quad \Lambda>\max \left(1, \frac{-1-2 \operatorname{Re} \mu}{d}\right),
\end{array}\right.
$$

la fonction

$$
R_{k}(x)=\frac{1}{2 i \pi} \int_{C_{2, k}} r_{k}(s) x^{-s} \mathrm{~d} s
$$

est bien définie et vérifie pour tout $\varepsilon>0$

$$
R_{k}(x)=O_{\varepsilon}\left(x^{-\frac{1}{2}-\frac{2-k}{d}+\varepsilon}\right),
$$

TOME $134-2006-\mathrm{N}^{\mathrm{O}} 3$ 
lorsque $x$ tend vers l'infini. Notons

$$
\begin{aligned}
& H_{k}(x)=\frac{1}{2 i \pi} \int_{C_{3, k}} f_{a, \nu_{k}}\left(d s+\beta_{k}\right) x^{-s} \mathrm{~d} s, \\
& G_{k}(x)=\frac{1}{2 i \pi} \int_{C_{3, k}} s f_{a, \nu_{k}}\left(d s+\beta_{k}\right) x^{-s} \mathrm{~d} s,
\end{aligned}
$$

où $C_{3, k}=C\left(T, c_{k}, \Lambda\right)$ avec $T, c_{k}, \Lambda$ vérifiant

$$
c_{k}<\frac{1}{2}-\frac{k}{d}, \quad T>\frac{2|\operatorname{Im} \mu|}{d}, \quad \Lambda>\max \left(0, c_{k}\right)
$$

Par définition, on a

$$
I_{k}(x)=c_{2, k} H_{k}(x)+c_{1, k} G_{k}(x)+R_{k}(x) .
$$

Nous allons à présent relier les intégrales $G_{k}$ et $H_{k}$ aux fonctions de Bessel $J_{\nu_{k}}$. Après changement de variable, si $C_{3, k}^{\prime}$ désigne l'image de $C_{3, k}$ par la transformation $s \mapsto d s+\beta_{k}$, on obtient en posant $\theta=2 \operatorname{Im} \mu / d$

$$
\begin{aligned}
H_{k}(x) & =\frac{1}{d} x^{\beta_{k} / d} \frac{1}{2 i \pi} \int_{C_{3, k}^{\prime}} f_{a, \nu_{k}}(z)\left(x^{1 / d}\right)^{-z} \mathrm{~d} z \\
& =\frac{1}{d} x^{-\frac{1}{2}+(k-1) / d+i \theta} J_{\nu_{k}}\left(a x^{1 / d}\right)=O\left(x^{-\frac{1}{2}+\frac{2 k-3}{2 d}}\right),
\end{aligned}
$$

d'après (8). D'autre part, $G_{k}(x)=-x H_{k}^{\prime}(x)$ donc on a

$$
\begin{aligned}
G_{k}(x)= & -\frac{1}{d}\left(-\frac{1}{2}+\frac{k-1}{d}+i \theta\right) x^{-\frac{1}{2}+\frac{k-1}{d}+i \theta} J_{\nu_{k}}\left(a x^{1 / d}\right) \\
& -\frac{a}{d^{2}} x^{-\frac{1}{2}+\frac{k}{d}+i \theta} J_{\nu_{k}}^{\prime}\left(a x^{1 / d}\right) \\
= &
\end{aligned}
$$

d'après (8) et l'équation différentielle $x J_{\nu}^{\prime}(x)-\nu J_{\nu}(x)=-x J_{\nu+1}(x)$ (formule (4), § 3.2 de [12]).

Finalement, on a $I_{k}(x)=O\left(x^{-\frac{1}{2}-\frac{1}{2 d}+\frac{k-1}{d}}\right)$, donc puisque $d \geq 1$, on a

$$
\varphi(x)=O\left(x^{-\frac{1}{2}\left(1+\frac{1}{d}\right)}\right) \text { et } \Phi(x)=O\left(x^{-\frac{1}{2}\left(1+\frac{1}{d}\right)}\right) .
$$

La fonction $\Phi$ appartient donc à l'espace $L^{2}(0,+\infty)$ et donc d'après la proposition 4.1, l'opérateur $U_{F}$ agit sur une fonction $f$ de $L^{2}(0,+\infty)$ de la manière suivante :

$$
U_{F} f(t):=\lim _{T \rightarrow+\infty} \int_{1 / T}^{T} f(v) \frac{\mathrm{d}}{\mathrm{d} v} \varphi\left(\frac{t}{v}\right) \mathrm{d} v .
$$

BULletin DE LA SOCIÉtÉ MATHÉMATIQUE DE FRANCE 


\section{Choix de vecteurs orthogonaux}

6.1. Étude formelle. - Nous minorerons $D(\lambda)$ par la norme de la projection orthogonale de $\chi$ sur un sous-espace orthogonal à $B_{F}^{\lambda}$. Nous chercherons donc des vecteurs orthogonaux à $B_{F}^{\lambda}$.

On supposera désormais que la fonction $F$ ne s'annule pas dans le demiplan $\operatorname{Re} s>\frac{1}{2}$.

En effet, si la fonction $F$ s'annule dans ce demi-plan, alors la distance $D(\lambda)$ ne tend pas vers 0 d'après le théorème 1 et la minoration du théorème 2 est alors évidente.

Notations 5. - Soient $w$ un nombre complexe et $\ell$ un entier naturel. On définit la fonction $\psi_{w, \ell}$ sur $\mathbb{R}^{+*}$ par

$$
\psi_{w, \ell}(t)=\left(\log \frac{1}{t}\right)^{\ell} t^{-w} \chi(t) .
$$

Notons que la fonction $\psi_{w, \ell}$ appartient à $L^{2}(0,+\infty)$ si et seulement si $\operatorname{Re} w<\frac{1}{2}$.

Rappelons que pour $\frac{1}{2}<\sigma<1$,

$$
\mathcal{M} \Psi_{F}^{(1)}(s)=-\frac{F(s)}{s} .
$$

Si on considère le produit scalaire hilbertien de l'espace $L^{2}(0,+\infty)$, en posant

$$
f:=\sum_{k=1}^{n} c_{k} \sqrt{\alpha_{k}} D_{\alpha_{k}} \Psi_{F}^{(1)} \in \mathcal{B}_{F}{ }^{\lambda}
$$

on a donc formellement pour tout $\ell \in \mathbb{N}$ et $\operatorname{Re} s=\frac{1}{2}$ :

$$
\left\langle f, \psi_{s, \ell}\right\rangle=\int_{0}^{+\infty} f(t) \overline{\psi_{s, \ell}(t)} \mathrm{d} t=\left(-\frac{\mathrm{d}}{\mathrm{d} s}\right)^{\ell} \sum_{k=1}^{n} c_{k} \alpha_{k}^{s}\left(-\frac{F(s)}{s}\right) .
$$

Le second membre est nul pour $s=\rho$ où $\rho$ est un zéro de $F$ et pour tout $\ell \leq m_{\rho}-1$ où $m_{\rho}$ est la multiplicité du zéro $\rho$ de $F$. Si les vecteurs $\psi_{\rho, \ell}$ appartenaient à l'espace $L^{2}(0,+\infty)$, on aurait donc $m_{\rho}$ vecteurs orthogonaux à l'espace $\mathcal{B}_{F}$ pour chaque zéro $\rho$ de $F$. Nous allons donc modifier ces vecteurs afin d'obtenir des vecteurs de $L^{2}(0,+\infty)$.

Avant tout, commençons par modifier la fonction $f$ afin d'obtenir une fonction de $L^{2}(0,1)$.

Définition 7. - - Si $m_{F}$ désigne l'ordre du pôle 1 de $F$, on définit la fonction $Z_{F}$ comme suit :

$$
Z_{F}(s)=\left(\frac{s-1}{s}\right)^{m_{F}}\left(-\frac{F(s)}{s}\right)
$$

TOME $134-2006-\mathrm{N}^{\mathrm{O}} 3$ 
- On note $P$ l'opérateur unitaire de symbole

$$
P(s):=\frac{s-1}{s} .
$$

- L'opérateur $P$ est l'inverse de l'opérateur $H-1$.

- Les fonctions $A_{F}$ et $\chi_{k}$ sont définies par

$$
A_{F}:=P^{m_{F}} \Psi_{F}^{(1)} \quad \text { et } \quad \chi_{k}:=P^{k} \chi .
$$

On a la propriété suivante :

Proposition 6.1. - La fonction $A_{F}$ appartient à $L^{2}(0,1)$ et pour $\operatorname{Re} s>\frac{1}{2}$,

$$
\mathcal{M} A_{F}(s)=Z_{F}(s) \text {. }
$$

Démonstration. - Pour $\frac{1}{2}<\sigma<1$,

$$
\mathcal{M} A_{F}(s)=Z_{F}(s) \text {. }
$$

Or la fonction $Z_{F}$ est une fonction de l'espace de Hardy du demi-plan $\operatorname{Re} s>\frac{1}{2}$ donc d'après le théorème de Paley-Wiener, $A_{F}$ est une fonction de $L^{2}(0,1)$ et l'égalité $\mathcal{M} A_{F}(s)=Z_{F}(s)$ est valable pour $\sigma>\frac{1}{2}$.

Remarquons de plus que comme l'opérateur $P$ est unitaire, la distance $D_{\lambda}$ que l'on cherche est égale à la distance entre la fonction $\chi_{m_{F}}$ et l'espace $C_{F}^{\lambda}$ suivant :

DÉfinition 8. - On appellera $C_{F}^{\lambda}$ le sous-espace de $L^{2}(0,1)$ suivant :

$$
C_{F}^{\lambda}=\left\{t \mapsto \sum_{k=1}^{n} c_{k} \sqrt{\alpha_{k}} D_{\alpha_{k}} A_{F}(t) ; n \in \mathbb{N}, \forall k \in[1, n], \lambda \leq \alpha_{k} \leq 1\right\}
$$

Cette première opération nous a permis de remplacer $\mathcal{B}_{F}^{\lambda}$ par un sous-espace de $L^{2}(0,1)$.

Avec ces notations, pour

$$
f(t):=\sum_{k=1}^{n} c_{k} \sqrt{\alpha_{k}} D_{\alpha_{k}} A_{F}(t) \in C_{F}^{\lambda}
$$

on a donc formellement pour $\operatorname{Re} s=\frac{1}{2}$ et pour tout $\ell \in \mathbb{N}$

$$
\left\langle f, \psi_{s, \ell}\right\rangle=\left(-\frac{\mathrm{d}}{\mathrm{d} s}\right)^{\ell} \sum_{k=1}^{n} c_{k} \alpha_{k}^{s} Z_{F}(s) .
$$

BULletin DE LA SOCiÉtÉ MATHÉmATiQUE DE FRANCE 


\subsection{Modification des vecteurs : conservation de l'orthogonalité}

Nous allons à présent modifier les vecteurs $\psi_{s, \ell}$ en leur appliquant des opérateurs.

DÉfinition 9. - On définit l'opérateur $V_{F}$, opérateur de phase associé à la fonction $A_{F}$. On a donc

$$
V_{F}\left(A_{F}\right)=J\left(A_{F}\right),
$$

et le symbole associé à $V_{F}$ est la fonction

$$
V_{F}(s):=\left(\frac{s}{1-s}\right)^{2 m_{F}} U_{F}(s) .
$$

DÉfinition 10. - Soit $\lambda$ un réel vérifiant $0<\lambda<1$. Supposons que la limite lorsque le complexe $w$ de partie réelle strictement inférieure à $\frac{1}{2}$ tend vers $s=\frac{1}{2}+i \tau$ de

$$
V_{F}^{-1} Q_{\lambda} V_{F}\left(\psi_{w, \ell}\right)
$$

existe dans $L^{2}(0,+\infty)$. On note alors $Y_{s, \ell}^{\lambda, F}$ cette limite.

En cas d'existence de la limite, calculons le produit hilbertien d'une fonction appartenant à l'espace $C_{\lambda}^{F}$ avec la fonction $Y_{s, \ell}^{\lambda, F}$. Nous allons montrer que l'application des opérateurs conserve le produit hilbertien.

Proposition 6.2. - Si la limite qui définit $Y_{s, \ell}^{\lambda, F}$ existe, alors pour toute fonction $f \in C_{F}^{\lambda}$ définie par

on $a$

$$
f:=\sum_{k=1}^{n} c_{k} \sqrt{\alpha_{k}} D_{\alpha_{k}} A_{F}
$$

$$
\left\langle f, Y_{s, \ell}^{\lambda, F}\right\rangle=\left(-\frac{\mathrm{d}}{\mathrm{d} s}\right)^{\ell}\left(\sum_{k=1}^{n} c_{k} \alpha_{k}^{s} Z_{F}(s)\right) .
$$

Démonstration. - Soit $w$ un complexe vérifiant $\operatorname{Re} w<\frac{1}{2}$. On a

$$
\begin{aligned}
\left\langle V_{F}^{-1} Q_{\lambda} V_{F}\left(\psi_{w, \ell}\right), f\right\rangle & =\sum_{k=1}^{n} \bar{c}_{k} \sqrt{\alpha_{k}}\left\langle V_{F}^{-1} Q_{\lambda} V_{F}\left(\psi_{w, \ell}\right), D_{\alpha_{k}} A_{F}\right\rangle \\
& =\sum_{k=1}^{n} \bar{c}_{k} \sqrt{\alpha_{k}}\left\langle V_{F}\left(\psi_{w, \ell}\right), Q_{\lambda} D_{\alpha_{k}} V_{F} A_{F}\right\rangle
\end{aligned}
$$

en utilisant successivement le fait que $V_{F}$ est unitaire, invariant et $Q_{\lambda}$ autoadjoint. Observons à présent que $V_{F} A_{F}=J A_{F}$. On obtient donc

$$
\begin{aligned}
\left\langle V_{F}^{-1} Q_{\lambda} V_{F}\left(\psi_{w, \ell}\right), f\right\rangle & =\sum_{k=1}^{n} \bar{c}_{k} \sqrt{\alpha_{k}}\left\langle V_{F}\left(\psi_{w, \ell}\right), D_{\alpha_{k}} V_{F} A_{F}\right\rangle \\
& =\sum_{k=1}^{n} \bar{c}_{k} \sqrt{\alpha_{k}}\left\langle\psi_{w, \ell}, D_{\alpha_{k}} A_{F}\right\rangle
\end{aligned}
$$

TOME $134-2006-\mathrm{N}^{\mathrm{O}} 3$ 


$$
\begin{aligned}
& =\left(\frac{\mathrm{d}}{\mathrm{d} w}\right)^{\ell} \sum_{k=1}^{n} \bar{c}_{k} \sqrt{\alpha_{k}}\left\langle D_{\alpha_{k}^{-1}} \psi_{w, 0}, A_{F}\right\rangle \\
& =\left(\frac{\mathrm{d}}{\mathrm{d} w}\right)^{\ell} \sum_{k=1}^{n} \bar{c}_{k} \alpha_{k}^{-w} \int_{0}^{1} t^{-w} \overline{A_{F}(t)} \mathrm{d} t .
\end{aligned}
$$

Prenons maintenant la limite lorsque $w$ tend vers $s$ puis le conjugué de notre expression. On obtient

$$
\left\langle f, Y_{s, \ell}^{\lambda, F}\right\rangle=\left(-\frac{\mathrm{d}}{\mathrm{d} s}\right)^{\ell}\left(\sum_{k=1}^{n} c_{k} \alpha_{k}^{s} Z_{F}(s)\right)
$$

6.3. Convergence dans $\boldsymbol{L}^{\mathbf{2}}(\mathbf{0},+\infty)$. - Nous venons de voir qu'en cas d'existence de la limite, les vecteurs que l'on a choisis conservent le produit hilbertien formel. Nous allons maintenant montrer que le choix des opérateurs de phase $U_{F}$ et $V_{F}$ garantie l'existence de cette limite. En effet, c'est le comportement de $\psi_{w, \ell}$ au voisinage de 0 qui entraine la divergence du produit scalaire formel. Les opérateurs $U_{F}$ et $V_{F}$ conservent la localisation de ce problème et l'application du projecteur $Q_{\lambda}$ le supprime, et permet ainsi d'obtenir une limite dans $L^{2}(0,+\infty)$.

$\mathrm{Au}$ vu de (18), il est naturel d'introduire la fonction suivante.

DÉfinition 11. - Soient $k \in \mathbb{N}$ et $w \in \mathbb{C}$ tel que $0<\operatorname{Re} w<\frac{1}{2}\left(1+\frac{1}{d}\right)$. On définit, si elle existe, la limite ponctuelle de la fonction de $\mathbb{R}^{+*}$ suivante :

$$
\phi_{w, k}(t):=\lim _{\eta \rightarrow 0} \int_{\eta}^{1}\left(\log \left(\frac{1}{v}\right)\right)^{k} v^{-w} \frac{\mathrm{d}}{\mathrm{d} v} U_{F} \chi\left(\frac{t}{v}\right) \mathrm{d} v .
$$

Remarque 3. - D'après (18), on a déjà montré l'existence de cette limite pour $\operatorname{Re} w<\frac{1}{2}$ et l'on sait de plus que dans ce cas $U_{F} \psi_{w, \ell}=\phi_{w, \ell}$.

Nous allons montrer l'existence de $\phi_{w, k}(t)$ pour $0<\operatorname{Re} w<\frac{1}{2}\left(1+\frac{1}{d}\right)$ et nous en donnerons une majoration uniforme en $w$ appartenant à un compact $K$ de la bande verticale $0<\operatorname{Re} w<\frac{1}{2}\left(1+\frac{1}{d}\right)$.

Proposition 6.3. - Pour tout $t>0$, tout $k \in \mathbb{N}$ et tout complexe $w$ vérifiant $0<\operatorname{Re} w<\frac{1}{2}\left(1+\frac{1}{d}\right)$, la limite définissant $\phi_{w, k}(t)$ existe. Pour tout $t>0$ fixé, cette limite est holomorphe en $w$. Si $\varepsilon$ est un réel strictement positif et si $w$ appartient à un compact $K$ de la bande verticale $\varepsilon \leq \operatorname{Re} w<\frac{1}{2}\left(1+\frac{1}{d}\right)$, alors on a uniformément par rapport à $w$ les majorations

$$
\phi_{w, k}(t)= \begin{cases}O\left(t^{-\frac{1}{2}\left(1+\frac{1}{d}\right)}\right), & \text { si } t \geq 1, \\ \delta_{0, k} U_{F} \chi(t)+\left(\frac{\mathrm{d}}{\mathrm{d} w}\right)^{k}\left(U_{F}(w) x^{-w}\right)+O\left(t^{-\varepsilon}\right) & \text { si } 0<t \leq 1 .\end{cases}
$$


Démonstration. - Intégrons par parties l'intégrale définissant $\phi_{w, k}(t)$ :

$$
\begin{aligned}
& \int_{\eta}^{1}\left(\log \left(\frac{1}{v}\right)\right)^{k} v^{-w} \frac{\mathrm{d}}{\mathrm{d} v} U_{F} \chi\left(\frac{t}{v}\right) \mathrm{d} v {\left[\left(\log \left(\frac{1}{v}\right)\right)^{k} v^{-w} U_{F} \chi\left(\frac{t}{v}\right)\right]_{\eta}^{1} } \\
& \quad+\int_{\eta}^{1}\left(k+w \log \frac{1}{v}\right)\left(\log \left(\frac{1}{v}\right)\right)^{k-1} v^{-w-1} U_{F} \chi\left(\frac{t}{v}\right) \mathrm{d} v \\
&=\delta_{0, k} U_{F} \chi(t)-\left(\log \left(\frac{1}{\eta}\right)\right)^{k} \eta^{-w} U_{F} \chi\left(\frac{t}{\eta}\right) \\
& \quad+\int_{1}^{1 / \eta}(k+w \log u)(\log u)^{k-1} u^{w-1} U_{F} \chi(t u) \mathrm{d} u
\end{aligned}
$$

en effectuant le changement de variable $u=1 / v$ dans la dernière intégrale.

Lorsque $t$ tend vers l'infini, on a $U_{F} \chi(t)=O\left(t^{-\frac{1}{2}\left(1+\frac{1}{d}\right)}\right)$ d'après $(12)$; donc

$$
\left(\log \left(\frac{1}{\eta}\right)\right)^{k} \eta^{-w} U_{F} \chi\left(\frac{t}{\eta}\right) \ll t^{-\frac{1}{2}\left(1+\frac{1}{d}\right)}\left(\log \left(\frac{1}{\eta}\right)\right)^{k} \eta^{\frac{1}{2}\left(1+\frac{1}{d}\right)-\operatorname{Re} w}
$$

et si $\operatorname{Re} w<\frac{1}{2}\left(1+\frac{1}{d}\right)$, on a

$$
\lim _{\eta \rightarrow 0}\left(\log \left(\frac{1}{\eta}\right)\right)^{k} \eta^{-w} U_{F} \chi\left(\frac{t}{\eta}\right)=0 .
$$

- Si on suppose $t \geq 1$, on a pour $\operatorname{Re} w<\frac{1}{2}\left(1+\frac{1}{d}\right)$,

$$
\begin{aligned}
\int_{1}^{+\infty}(k+w \log u)(\log u)^{k-1} u^{w-1} U_{F} \chi(t u) \mathrm{d} u \\
=O\left(\int_{1}^{+\infty}(k+w \log u)(\log u)^{k-1} u^{\operatorname{Re} w-1}(t u)^{-\frac{1}{2}\left(\frac{1}{d}+1\right)} \mathrm{d} u\right) \\
\quad=O\left(t^{-\frac{1}{2}\left(\frac{1}{d}+1\right)}\right)
\end{aligned}
$$

La limite définissant $\phi_{w, k}(t)$ existe donc bien. Pour tout $t \geq 1$ fixé, cette limite est holomorphe en $w$. Si $w$ appartient à un compact $K$ de la bande verticale $0<\operatorname{Re} w<\frac{1}{2}\left(1+\frac{1}{d}\right)$ et si $t \geq 1$, alors on a uniformément par rapport à $w$ la majoration

$$
\phi_{w, k}(t)=O\left(t^{-\frac{1}{2}\left(1+\frac{1}{d}\right)}\right) .
$$

- Supposons maintenant $0<t<1$. Remarquons tout d'abord que, pour tout $k \in \mathbb{N}$, on a

$$
\left(\frac{\mathrm{d}}{\mathrm{d} w}\right)^{k}\left(w u^{w-1}\right)=(k+w \log u)(\log u)^{k-1} u^{w-1} .
$$

TOME $134-2006-\mathrm{N}^{\mathrm{O}} 3$ 
Cette formule se montre facilement par récurrence sur $k$. Comme l'intégrale converge uniformément par rapport à $w$ dans un compact de la bande verticale $0<\operatorname{Re} w<\frac{1}{2}\left(1+\frac{1}{d}\right)$, on a donc

$$
\begin{aligned}
& \int_{0}^{+\infty}(k+w \log u)(\log u)^{k-1} u^{w-1} U_{F} \chi(t u) \mathrm{d} u \\
&=\left(\frac{\mathrm{d}}{\mathrm{d} w}\right)^{k}\left(w \int_{0}^{+\infty} u^{w-1} U_{F} \chi(t u) \mathrm{d} u\right) .
\end{aligned}
$$

En posant $v=t u$, on a pour $0<\operatorname{Re} w<\frac{1}{2}\left(1+\frac{1}{d}\right)$,

On a donc finalement

$$
\begin{aligned}
\int_{0}^{+\infty} u^{w-1} U_{F} \chi(t u) \mathrm{d} u & =t^{-w} \int_{0}^{+\infty} v^{w-1} U_{F} \chi(v) \mathrm{d} v \\
& =t^{-w} \mathcal{M}\left(U_{F} \chi\right)(w)=t^{-w} \frac{U_{F}(w)}{w}
\end{aligned}
$$

$$
\int_{0}^{+\infty}(k+w \log u)(\log u)^{k-1} u^{w-1} U_{F} \chi(t u) \mathrm{d} u=\left(\frac{\mathrm{d}}{\mathrm{d} w}\right)^{k}\left(t^{-w} U_{F}(w)\right) .
$$

Examinons à présent l'intégrale entre 0 et 1 . Soit $\varepsilon>0$. D'après la proposition 4 , on a $U_{F} \chi(t)=O\left(t^{-\varepsilon}\right)$ lorsque $t$ tend vers 0 . On a donc

$$
\begin{aligned}
\int_{0}^{1}(k+w \log u) & (\log u)^{k-1} u^{w-1} U_{F} \chi(t u) \mathrm{d} u \\
& =O\left(t^{-\varepsilon} \int_{0}^{1}(k+w \log u)(\log u)^{k-1} u^{\operatorname{Re} w-\varepsilon-1} \mathrm{~d} u\right) .
\end{aligned}
$$

Si $\operatorname{Re} w>\varepsilon$, on a donc

$$
\int_{0}^{1}(k+w \log u)(\log u)^{k-1} u^{w-1} U_{F} \chi(t u) \mathrm{d} u=O\left(t^{-\varepsilon}\right) .
$$

On a donc uniformément pour $w$ dans un compact $K$ de la bande verticale $\varepsilon<\operatorname{Re} w<\frac{1}{2}\left(1+\frac{1}{d}\right)$

si $0<t \leq 1$.

$$
\phi_{w, k}(t)=\delta_{0, k} U_{F} \chi(t)+\left(\frac{\mathrm{d}}{\mathrm{d} w}\right)^{k}\left(U_{F}(w) t^{-w}\right)+O\left(t^{-\varepsilon}\right)
$$

Nous allons par la suite travailler non avec $U_{F} \psi_{w, k}$ mais avec $V_{F} \psi_{w, k}$. Nous allons donc être amenés à appliquer l'opérateur de Hardy $H$ plusieurs fois à la fonction $\phi_{w, k}$. Nous aurons pour cela besoin de la proposition suivante.

Proposition 6.4. - Soit $k \in \mathbb{N}$. Pour chaque complexe $w$ de la bande verticale $\left(0<\operatorname{Re} w<\frac{1}{2}\left(1+\frac{1}{d}\right)\right)$, l'opérateur de Hardy

$$
H: f(t) \longmapsto \frac{1}{t}\left(\int_{0}^{t} f(v) \mathrm{d} v\right)
$$

BULletin DE LA SOCIÉtÉ MATHÉMATIQUE DE FRANCE 
peut être appliqué autant de fois que l'on veut à la fonction $\phi_{w, k}$. Si $L \in \mathbb{N}$, on a de plus pour $t \geq 1$ uniformément par rapport à $w$ dans la bande verticale $\left(0<\operatorname{Re} w<\frac{1}{2}\left(1+\frac{1}{d}\right)\right)$, la majoration

$$
H^{L}\left(\phi_{w, k}\right)(t)=O\left(1+\delta_{d, 1} \log t\right)^{L} t^{-\frac{1}{2}\left(1+\frac{1}{d}\right)},
$$

et pour tout $\varepsilon>0$, pour $0<t<1$, uniformément par rapport à $w$ dans la bande verticale $\left(\varepsilon<\operatorname{Re} w<\frac{1}{2}\left(1+\frac{1}{d}\right)\right)$, la majoration

$$
H^{L}\left(\phi_{w, k}\right)(t)=\frac{1}{(1-w)^{L}}\left(\frac{\mathrm{d}}{\mathrm{d} w}\right)^{k}\left(U_{F}(w) t^{-w}\right)+O\left(t^{-\varepsilon}\right) .
$$

Démonstration. - Soit $k \in \mathbb{N}$. Remarquons dans un premier temps que pour $L \in \mathbb{N}$ et $t>1$, on a

$$
\int_{1}^{t} \frac{1}{v}(1+\log v)^{L} \mathrm{~d} v=\frac{1}{L+1}\left((1+\log t)^{L+1}-1\right)
$$

Nous allons maintenant démontrer la proposition par récurrence sur $L$.

- Pour $L=0$, c'est la proposition 6.3.

- Supposons la proposition vérifiée au rang $L-1$.

Supposons dans un premier temps $t \geq 1$ et $0<\operatorname{Re} w<\frac{1}{2}\left(1+\frac{1}{d}\right)$.

$$
\begin{aligned}
& H^{L}\left(\phi_{w, k}\right)(t)=\frac{1}{t} \int_{0}^{t} H^{L-1}\left(\phi_{w, k}\right)(v) \mathrm{d} v \\
& \quad=\frac{1}{t}\left(\int_{0}^{1} H^{L-1}\left(\phi_{w, k}\right)(v) \mathrm{d} v+\int_{1}^{t} O\left(1+\delta_{d, 1} \log v\right)^{L-1} v^{-\frac{1}{2}\left(1+\frac{1}{d}\right)} \mathrm{d} v\right) .
\end{aligned}
$$

Si $d=1$, on a donc

$$
H^{L}\left(\phi_{w, k}\right)(t)=O\left(\frac{1}{t}\right)+O\left(\frac{1}{t} \int_{1}^{t}(1+\log v)^{L-1} v^{-1} \mathrm{~d} v\right)=O\left(\frac{1}{t}(1+\log t)^{L}\right)
$$

et si $d>1$, on a

$$
H^{L}\left(\phi_{w, k}\right)(t)=\frac{1}{t} O\left(1+\int_{1}^{t} v^{-\frac{1}{2}\left(1+\frac{1}{d}\right)} \mathrm{d} v\right)=O\left(t^{-\frac{1}{2}\left(1+\frac{1}{d}\right)}\right) .
$$

Supposons maintenant $\varepsilon>0,0<t<1$ et $\left.\varepsilon<\operatorname{Re} w<\frac{1}{2}\left(1+\frac{1}{d}\right)\right)$; on a alors

$$
\begin{aligned}
H^{L}\left(\phi_{w, k}\right)(t) & =\frac{1}{t} \int_{0}^{t} H^{L-1}\left(\phi_{w, k}\right)(v) \mathrm{d} v \\
& =\frac{1}{t} \int_{0}^{t}\left(\frac{1}{(1-w)^{L-1}}\left(\frac{\mathrm{d}}{\mathrm{d} w}\right)^{k}\left(U_{F}(w) v^{-w}\right)+O\left(v^{-\varepsilon}\right)\right) \mathrm{d} v \\
& =\frac{1}{(1-w)^{L-1}}\left(\frac{\mathrm{d}}{\mathrm{d} w}\right)^{k}\left(U_{F}(w) \frac{1}{t} \int_{0}^{t} v^{-w} \mathrm{~d} v\right)+O\left(t^{-\varepsilon}\right) \\
& =\frac{1}{(1-w)^{L}}\left(\frac{\mathrm{d}}{\mathrm{d} w}\right)^{k}\left(U_{F}(w) t^{-w}\right)+O\left(t^{-\varepsilon}\right) .
\end{aligned}
$$

TOME $134-2006-\mathrm{N}^{\mathrm{O}} 3$ 
La propriété est donc vraie au rang $L$.

Il est clair, au vu des propositions 6.3 et 6.4, que le problème de convergence posé par les fonctions $H^{L}\left(\phi_{w, k}\right)$ reste localisé en zéro. L'application du projecteur $Q_{\lambda}$ nous permettra donc d'obtenir des fonctions de $L^{2}(0,+\infty)$. De plus, nous avons obtenu une majoration uniforme en $w$ de ces fonctions par une fonction de $L^{2}(0,+\infty)$. Grâce au théorème de Lebesgue, on peut déduire de cette étude l'existence des vecteurs $Y_{s, \ell}^{\lambda, F}$. On obtient également une évaluation de ces vecteurs.

Proposition 6.5. - Soient $\lambda>0, k \in \mathbb{N}$ et $s$ un complexe de partie réelle égale $\grave{a} \frac{1}{2}$. Alors $Y_{s, k}^{\lambda}$ existe et pour tout $\varepsilon>0$, on a

$$
V_{F}\left(Y_{s, k}^{\lambda}\right)(t)= \begin{cases}O\left(\left(1+\delta_{1, d} \log t\right)^{2 m_{F}} t^{-\frac{1}{2}\left(1+\frac{1}{d}\right)}\right) & \text { si } t \geq 1, \\ \left(\frac{\mathrm{d}}{\mathrm{d} s}\right)^{k}\left(V_{F}(s) t^{-s}\right)+O_{\varepsilon}\left(t^{-\varepsilon}\right) & \text { si } \lambda<t \leq 1, \\ 0 & \text { si } 0<t<\lambda .\end{cases}
$$

\section{Démonstration du théorème}

7.1. Calculs de produits hilbertiens. - Grâce à l'étude précédente, nous pouvons introduire des vecteurs orthogonaux à l'espace $C_{F}^{\lambda}$.

DÉfinition 12. - Soit $0<\lambda<1$. À chaque zéro $\rho$ de la fonction $F$ de multiplicité $m_{\rho}$ et à chaque entier $0 \leq k<m_{\rho}$, on associe le vecteur

$$
X_{\rho, k}^{\lambda, F}:=\left(\log \frac{1}{\lambda}\right)^{-\frac{1}{2}-k} Y_{\rho, k}^{\lambda, F} .
$$

Soit $R$ un ensemble non-vide de zéros de $F$. On a montré que $D(\lambda)$ était la distance dans l'espace de Hilbert $L^{2}(0,+\infty)$ entre $\chi_{m_{F}}$ et $C_{F}^{\lambda}$ et que les vecteurs $X_{\rho, k}^{\lambda, F}$ pour $0 \leq k<m_{\rho}$ sont perpendiculaires à $C_{F}^{\lambda}$. La distance $D(\lambda)$ est donc minorée par la norme de la projection orthogonale de $\chi_{m_{F}}$ sur l'espace vectoriel $H_{R}$ engendré par les vecteurs $X_{\rho, k}^{\lambda, F}, 0 \leq k<m_{\rho}, \rho \in R$. Cette norme est égale à la racine carrée du produit matriciel

$$
\left[\left\langle\chi_{m_{F}}, X_{\rho, k}^{\lambda, F}\right\rangle\right] \operatorname{Gram}\left(X_{\rho, k}^{\lambda, F}\right)^{-1}\left[\left\langle X_{\rho, k}^{\lambda, F}, \chi_{m_{F}}\right\rangle\right]^{*},
$$

où $\operatorname{Gram}\left(X_{\rho, k}^{\lambda, F}\right)$ est la matrice $\operatorname{Gram}_{\lambda, F}$ des vecteurs $X_{\rho, k}^{\lambda, F}, 0 \leq k<m_{\rho}, \rho \in R$. Il nous faut donc calculer les produits hilbertiens qui interviennent dans cette expression.

Proposition 7.1. - Si $\rho_{1}$ et $\rho_{2}$ sont deux zéros distincts de la fonction $F$, pour tout entier $0 \leq k<m_{\rho_{1}}$ et tout entier $0 \leq \ell<m_{\rho_{2}}$, on a

$$
\lim _{\lambda \rightarrow 0}\left\langle X_{\rho_{1}, k}^{\lambda, F}, X_{\rho_{2}, \ell}^{\lambda, F}\right\rangle=0 .
$$

BULletin DE LA SOCIÉtÉ MATHÉMATIQUE DE FRANCE 
Si $\rho$ est un zéro de la fonction $F$, pour tout entiers $0 \leq k, \ell<m_{\rho}$, on a

$$
\lim _{\lambda \rightarrow 0}\left\langle X_{\rho, k}^{\lambda, F}, X_{\rho, \ell}^{\lambda, F}\right\rangle=\frac{1}{k+\ell+1}
$$

Démonstration. - Une simple intégration par parties nous permet de remarquer que pour $\operatorname{Re} s_{1}=\operatorname{Re} s_{2}=\frac{1}{2}$, on a lorsque $\lambda$ tend vers 0 :

$$
\int_{\lambda}^{1}\left(\log \frac{1}{t}\right)^{j_{1}} t^{-s_{1}}\left(\log \frac{1}{t}\right)^{j_{2}} t^{-\left(1-s_{2}\right)} \mathrm{d} t= \begin{cases}O\left(\log \frac{1}{\lambda}\right)^{j_{1}+j_{2}} & \text { si } s_{1} \neq s_{2} \\ \frac{1}{j_{1}+j_{2}+1}\left(\log \frac{1}{\lambda}\right)^{j_{1}+j_{2}+1} & \text { si } s_{1}=s_{2}\end{cases}
$$

L'opérateur $V_{F}$ est unitaire; on a donc

$$
\left\langle X_{\rho_{1}, k}^{\lambda, F}, X_{\rho_{2}, \ell}^{\lambda, F}\right\rangle=\left\langle V_{F} X_{\rho_{1}, k}^{\lambda, F}, V_{F} X_{\rho_{2}, \ell}^{\lambda, F}\right\rangle=\left(\log \frac{1}{\lambda}\right)^{-1-k-\ell}\left\langle V_{F} Y_{\rho_{1}, k}^{\lambda, F}, V_{F} Y_{\rho_{2}, \ell}^{\lambda, F}\right\rangle .
$$

Utilisons maintenant la proposition 6.5 , on obtient

$$
\begin{aligned}
&\left\langle X_{\rho_{1}, k}^{\lambda, F}, X_{\rho_{2}, \ell}^{\lambda, F}\right\rangle \\
&=\left(\log \frac{1}{\lambda}\right)^{-1-k-\ell} \int_{0}^{+\infty} V_{F} Y_{\rho_{1}, k}^{\lambda, F}(t) \overline{V_{F} Y_{\rho_{2}, \ell}^{\lambda, F}}(t) \mathrm{d} t \\
&=\left(\log \frac{1}{\lambda}\right)^{-1-k-\ell}\left\{\int_{1}^{+\infty}\left(1+\delta_{1, d} \log t\right)^{4 m_{F}} t^{-\left(1+\frac{1}{d}\right)} \mathrm{d} t\right. \\
&+\int_{\lambda}^{1}\left(\left(\frac{\mathrm{d}}{\mathrm{d} s}\right)^{k}\left(\frac{\left.\left(V_{F}\left(\rho_{1}\right) t^{-\rho_{1}}\right)+O\left(t^{-\varepsilon}\right)\right)}{\left(\frac{\mathrm{d}}{\mathrm{d} s}\right)^{\ell}\left(V_{F}\left(\rho_{2}\right)\right) t^{-\rho_{2}}}+O\left(t^{-\varepsilon}\right)\right) \mathrm{d} t\right\} .
\end{aligned}
$$

Or on a vu que

$$
\begin{aligned}
\int_{\lambda}^{1}\left(\frac{\mathrm{d}}{\mathrm{d} s}\right)^{j_{1}} t^{-\rho_{1}} \overline{\left(\frac{\mathrm{d}}{\mathrm{d} s}\right)^{\ell} t^{-\rho_{2}}} \mathrm{~d} t & =\int_{\lambda}^{1}\left(\log \frac{1}{t}\right)^{j_{1}} t^{-\rho_{1}}\left(\log \frac{1}{t}\right)^{j_{2}} t^{-\left(1-\rho_{2}\right)} \mathrm{d} t \\
& = \begin{cases}O\left(\log \frac{1}{\lambda}\right)^{j_{1}+j_{2}} & \text { si } \rho_{1} \neq \rho_{2} \\
\frac{1}{j_{1}+j_{2}+1}\left(\log \frac{1}{\lambda}\right)^{j_{1}+j_{2}+1} & \text { si } \rho_{1}=\rho_{2}\end{cases}
\end{aligned}
$$

donc

$$
\lim _{\lambda \rightarrow 0}\left\langle X_{\rho_{1}, k}^{\lambda, F}, X_{\rho_{2}, \ell}^{\lambda, F}\right\rangle= \begin{cases}0 & \text { si } \rho_{1} \neq \rho_{2}, \\ \frac{1}{k+\ell+1} & \text { si } \rho_{1}=\rho_{2} .\end{cases}
$$

On a donc une expression simple de la matrice de Gram des vecteurs $X_{\rho, k}^{\lambda, F}$ lorsque $\lambda$ tend vers 0 . En effet, on vient de montrer que cette matrice converge vers une matrice diagonale par blocs.

Reste à étudier le produit hilbertien d'un vecteur $X_{\rho, k}^{\lambda, F}$ avec le vecteur $\chi_{m_{F}}$.

TOME $134-2006-\mathrm{N}^{\mathrm{O}} 3$ 
Proposition 7.2. - Si $\rho$ est un zéro de la fonction $F$, pour tout entier $0 \leq k<m_{\rho}$, on $a$

$$
\sqrt{\log \left(\frac{1}{\lambda}\right)} \lim _{\lambda \rightarrow 0}\left\langle\chi_{m_{F}}, X_{\rho, k}^{\lambda, F}\right\rangle= \begin{cases}0 & \text { si } k \geq 1 \\ \frac{1}{\rho}\left(1-\frac{1}{\rho}\right)^{m_{F}} & \text { si } k=0\end{cases}
$$

Démonstration. - Remarquons que les opérateurs $P$ et $1-H$ sont inverses l'un de l'autre. Comme $\chi_{m_{F}}=P^{m_{F}} \chi$, on a donc

$$
(1-H)^{m_{F}} \chi_{m_{F}}=\chi \text {. }
$$

Soient $\rho$ un zéro de la fonction $F$ et $k$ un entier vérifiant $0 \leq k<m_{\rho}$. Les opérateurs invariants $U_{F}$ et $1-H$ commutent; on a donc

$$
V_{F} \chi_{m_{F}}=(1-H)^{2 m_{F}} U_{F} \chi_{m_{F}}=(1-H)^{m_{F}} U_{F} \chi
$$

et donc

$$
\begin{aligned}
\sqrt{\log \left(\frac{1}{\lambda}\right)}\left\langle\chi_{m_{F}}, X_{\rho, k}^{\lambda, F}\right\rangle & =\sqrt{\log \left(\frac{1}{\lambda}\right)}\left\langle V_{F} \chi_{m_{F}}, V_{F} X_{\rho, k}^{\lambda, F}\right\rangle \\
& =\left(\log \frac{1}{\lambda}\right)^{-k}\left\langle(1-H)^{m_{F}} U_{F} \chi, V_{F} Y_{\rho, k}^{\lambda, F}\right\rangle
\end{aligned}
$$

Pour tout $\varepsilon>0$, lorsque $t$ tend vers 0 , on a

$$
U_{F} \chi(t)=O_{\varepsilon}\left(t^{-\varepsilon}\right)
$$

et, lorsque $t$ tend vers l'infini, on a

$$
U_{F} \chi(t)=O\left(t^{-\frac{1}{2}\left(1+\frac{1}{d}\right)}\right) .
$$

On a donc les mêmes majorations pour $(1-H)^{m_{F}} U_{F} \chi$.

D'autre part, d'après la proposition 6.5 , on a

$$
V_{F}\left(Y_{s, k}^{\lambda}\right)(t)= \begin{cases}O\left(\left(1+\delta_{1, d} \log t\right)^{\left.2 m_{F} t^{-\frac{1}{2}\left(1+\frac{1}{d}\right)}\right)}\right. & \text { si } t \geq 1, \\ \left(\frac{\mathrm{d}}{\mathrm{d} s}\right)^{k}\left(V_{F}(s) t^{-s}\right)+O_{\varepsilon}\left(t^{-\varepsilon}\right) & \text { si } \lambda<t \leq 1, \\ 0 & \text { si } 0<t<\lambda .\end{cases}
$$

On a donc en particulier

$$
\begin{aligned}
\int_{1}^{+\infty}(1-H)^{m_{F}} & U_{F} \chi(t) \overline{V_{F} Y_{\rho, k}^{\lambda, F}(t)} \mathrm{d} t \\
& =O\left(\int_{1}^{+\infty} t^{-\frac{1}{2}\left(1+\frac{1}{d}\right)}\left(1+\delta_{1, d} \log t\right)^{2 m_{F}} t^{-\frac{1}{2}\left(1+\frac{1}{d}\right)} \mathrm{d} t\right) \\
& =O\left(\int_{1}^{+\infty}\left(1+\delta_{1, d} \log t\right)^{2 m_{F}} t^{-\left(1+\frac{1}{d}\right)} \mathrm{d} t\right)=O(1)
\end{aligned}
$$

BULLETIN DE LA SOCIÉTÉ MATHÉMATIQUE DE FRANCE 
On a

$$
\begin{aligned}
\left\langle(1-H)^{m_{F}}\right. & \left.U_{F} \chi, V_{F} Y_{\rho, k}^{\lambda, F}\right\rangle \\
& =\int_{\lambda}^{1} t^{-\varepsilon} \overline{\left.\left(\frac{\mathrm{d}}{\mathrm{d} s}\right)^{k}\left(V_{F}(s) t^{-s}\right)+O_{\varepsilon}\left(t^{-\varepsilon}\right)\right)} \mathrm{d} t+O(1) \\
& =O\left(1+\int_{\lambda}^{1} t^{-\frac{1}{2}-\varepsilon}\left(\log \frac{1}{t}\right)^{k} \mathrm{~d} t\right)=O\left(\lambda^{\frac{1}{2}-\varepsilon}\left(\log \frac{1}{\lambda}\right)^{k}+1\right) .
\end{aligned}
$$

L'existence de la limite $\lim _{\lambda \rightarrow 0} \sqrt{\log (1 / \lambda)}\left\langle\chi_{m_{F}}, X_{\rho, k}^{\lambda, F}\right\rangle$ est donc établie et pour $k \geq 1$,

$$
\lim _{\lambda \rightarrow 0} \sqrt{\log \left(\frac{1}{\lambda}\right)}\left\langle\chi_{m_{F}}, X_{\rho, k}^{\lambda, F}\right\rangle=0 .
$$

Utilisons maintenant le caractère uniforme en $w$ de la limite dans la proposition 6.3. On a donc pour $k=0$,

$$
\begin{aligned}
\lim _{\lambda \rightarrow 0} \sqrt{\log \left(\frac{1}{\lambda}\right)}\left\langle\chi_{m_{F}},\right. & \left.X_{\rho, 0}^{\lambda, F}\right\rangle=\lim _{w \rightarrow \rho}\left\langle\chi_{m_{F}}, \psi_{w, 0}\right\rangle \\
& =\lim _{w \rightarrow \rho} \mathcal{M}\left(P^{m_{F}} \chi\right)(w)=P^{m_{F}}(\rho) \frac{1}{\rho}=\frac{1}{\rho}\left(1-\frac{1}{\rho}\right)^{m_{F}} .
\end{aligned}
$$

7.2. Conclusion. - Considérons à nouveau un ensemble $R$ non vide de zéros de $F$. Nous pouvons maintenant évaluer le produit matriciel (19) dont la racine carrée minore $D(\lambda)$.

D'après la proposition 7.1, la matrice de Gram converge lorsque $\lambda$ tend vers 0 vers une matrice diagonale par blocs. Il y a autant de blocs diagonaux que de zéros dans $R$ et le bloc correspondant au zéro $\rho$ est la matrice de Cauchy $\left(\frac{1}{i+j+1}\right)_{i, j \geq 0}$ de taille $m_{\rho} \times m_{\rho}$. On sait (cf. [8], vol. XII, p. 177) que l'élément en haut à gauche de la matrice inverse est $m_{\rho}^{2}$.

En utilisant en plus la proposition 7.2 qui donne une évaluation des matrices ligne et colonne intervenant dans le calcul lorsque $\lambda$ tend vers 0 , on obtient que la racine carrée de la projection orthogonale de $\chi_{m_{F}}$ sur l'espace vectoriel $H_{R}$ est asymptotiquement équivalente lorsque $\lambda$ tend vers 0 à $\sum_{\rho \in R} m_{\rho}^{2} /|\rho|^{2}$.

Ainsi, ceci étant vrai pour tout ensemble non vide de zéros de $F$, on a bien la minoration du théorème.

On a donc démontré le théorème 2.

\section{BIBLIOGRAPHIE}

[1] Báez-Duarte (L.) - A class of invariant unitary operators, Adv. in Math., t. 144 (1999), pp. 1-12.

TOME $134-2006-\mathrm{N}^{\mathrm{O}} 3$ 
[2] Báez-Duarte (L.), Balazard (M.), Landreau (B.) \& Saias (E.) Notes sur la fonction zeta de Riemann, 3, Adv. in Math., t. 149 (2000), pp. 130-144.

[3] BAlazard (M.) \& SAiAs (E.) - The Nyman-Beurling equivalent form for the Riemann hypothesis, Expo. Math., t. 18 (2000), pp. 131-138.

[4] Beurling (A.) - A closure problem related to the Riemann zeta-function, Proc. Nat. Acad. Sci., t. 41 (1955), pp. 312-314.

[5] Bourbaki (N.) - Éléments de mathématiques. Fonctions d'une variable réelle. Théorie élémentaire, Hermann, 1976.

[6] Burnol (J.-F.) - Quaternionic Gamma functions and their logarithmic derivatives as spectral functions, Math. Res. Lett., t. 8 (2001).

[7] _ A lower bound in an approximation problem involving the zeros of the Riemann zeta function, Adv. in Math., t. 170 (2002), pp. 56-70.

[8] CAuchy (A.) - Euvres complètes d'Augustin Cauchy, publiées sous la direction scientifique de l'Académie des Sciences, $\mathrm{II}^{\mathrm{e}}$ série, Gauthier-Villars, Paris.

[9] de Roton (A.) - Généralisation du critère de Beurling-Nyman pour l'hypothèse de Riemann généralisée, à paraître dans Acta Arithmetica.

[10] _ On the mean square of an error term for an extended Selberg's class, à paraître dans AMS Transactions.

[11] Selberg (A.) - Old and new conjectures and results about a class of Dirichlet series, in Proceedings of the Amalfi Conference on Analytic Number Theory (Maiori, 1989) (Salerno), Univ. Salerno, 1992, pp. 367-385.

[12] Watson (G. N.) - A treatise on the theory of Bessel functions, 2nd ed., Cambridge University Press, 1966. 